\title{
Two equations for linear recurrence sequences
}

\author{
by \\ V. LOSERT (Wien)
}

1. Introduction. Our main object will be two equations, the first of them being

$$
a u_{h}^{k}+b u_{n}^{l}+c u_{q}^{m}=0
$$

with given coefficients $a, b, c \in \mathbb{C} \backslash\{0\}$ and given exponents $k, l, m \in \mathbb{N}$ that are pairwise distinct. If $\left(u_{n}\right)_{n \in \mathbb{Z}}$ is a linear recurrence sequence, we will investigate the set of triples $(h, n, q) \in \mathbb{Z}^{3}$ solving this equation. The second equation is

$$
b u_{n}^{l}+c u_{q}^{m}=1
$$

with given coefficients $b, c \in \mathbb{C} \backslash\{0\}$ and given exponents $l, m \in \mathbb{N}$ satisfying $l \neq m$.

For a linear recurrence sequence $\left(u_{n}\right)_{n \in \mathbb{Z}}$, we denote by $\alpha_{1}, \ldots, \alpha_{r}$ the different roots of its companion polynomial (see [ST, p. 33], note that $\alpha_{i} \neq 0$ holds always). In [SS2], $r$ is called the rank of $\left(u_{n}\right)$. The sequence $\left(u_{n}\right)$ is called non-degenerate if $\alpha_{i} / \alpha_{j}$ is not a root of unity for $i \neq j$. Let $\mathcal{G}$ be the multiplicative group generated by $\alpha_{1}, \ldots, \alpha_{r}$. By $g$ we denote the (torsion free) rank of $\mathcal{G}$.

THEOREM 1. Let $\left(u_{n}\right)$ be a non-degenerate linear recurrence sequence with rank $r \geq 2$. Then, apart from the exceptional cases described below, equation (1.1) has only finitely many solutions $(h, n, q) \in \mathbb{Z}^{3}$.

The exceptional cases occur (after suitable reordering of the three terms of the sum) for: $m=1, l=2$ (implying $k>2$ ), $r=2$. Thus the exceptional equations are $a u_{h}^{k}+b u_{n}^{2}+c u_{q}=0$. The exceptions in the case of equation (1.2) (see below) generate exceptions for this equation by keeping $h$ fixed. This gives two classes of sequences with infinitely many solutions. For $k=3$, there are two further possibilities. First, if $\eta=\frac{4 b^{2}}{9 a c}$ is a root of unity, exceptions come from sequences $u_{n}=a_{2}\left( \pm \alpha^{n} / 2+\zeta^{n}\right)$ ( $\zeta$ a root of unity), when

2000 Mathematics Subject Classification: 11B37, 11D61. 
$a_{2}, \alpha, \zeta$ satisfy some additional algebraic equations: $\alpha / \zeta$ has to be an integer root of $2, a_{2}=-\frac{4 b}{3 a} \eta_{1}$, where $\eta_{1}^{2}=-1 / \eta$ and $\zeta$ is an integer root of $\eta_{1}$. Second, if $\eta=4\left(\frac{4 b^{2}}{9 a c}\right)^{5}$ is a root of unity, exceptions arise from sequences $u_{n}=a_{1}\left(\alpha^{-2 n} \pm \frac{1}{2} \zeta^{n} \alpha^{3 n}\right)$, with similar conditions for $a_{1}, \alpha, \zeta$ (e.g., $\alpha^{5} \zeta$ has to be an integer root of 2$)$. More details are given in Section 9 .

THEOREM 2. Let $\left(u_{n}\right)$ be a non-degenerate linear recurrence sequence with rank $r \geq 1$. Then, apart from the exceptional cases described below, equation (1.2) has only finitely many solutions $(n, q) \in \mathbb{Z}^{2}$.

The exceptional cases are (after suitable reordering of the two terms of the sum): $m=1, l=2, \eta=b /\left(2 c^{2}\right)$ is a root of unity, $r=2$. This leads to equations $2 \eta c^{2} u_{n}^{2}+c u_{q}=1$. The exceptional sequences are all simple, thus $u_{n}=a_{1} \alpha_{1}^{n}+a_{2} \alpha_{2}^{n}$. There are two possibilities for such a sequence $\left(u_{n}\right)$ so that the equation above has infinitely many solutions $(n, q)$. First, if there exists an integer $t$ such that $a_{1} / \alpha_{1}^{t}=a_{2} / \alpha_{2}^{t}=-1 /(2 \eta c)$ and $\alpha_{1} \alpha_{2}$ is an integer root of $\eta$. Prototypes (with $t=0$ ) are the sequences $w_{n}=-\frac{1}{2 \eta c}\left(\alpha^{n}+\left(\eta_{1} \alpha\right)^{-n}\right)$, where $\eta_{1}$ denotes any integer root of $\eta$. Second, if there exists an integer $t$ such that $a_{1}^{3} / \alpha_{1}^{t}=a_{2}^{3} / \alpha_{2}^{t}=-1 /\left(8 \eta c^{3}\right)$ and $\alpha_{1} \alpha_{2}$ is an integer root of $4 c^{2} \eta a_{1} a_{2}$. Prototypes (with $t=0$ ) are the sequences $\widetilde{w}_{n}=$ $-\frac{1}{2 \eta_{2} c}\left(\widetilde{\alpha}^{n}+\eta_{3}\left(\eta_{4} \widetilde{\alpha}\right)^{-n}\right)$, where $\eta_{2}, \eta_{3}, \eta_{4}$ are any numbers such that $\eta_{2}^{3}=\eta$, $\eta_{3}^{3}=1$ and $\eta_{4}$ is an integer root of $\eta_{2} \eta_{3}$. In all the exceptions $\alpha_{1} \alpha_{2}$ is a root of unity. For $\eta= \pm 1$, the two cases overlap. More details are given in Section 8 .

In all these exceptions (for Theorems 1 and 2) the polynomials $f_{i}$ are constant (in the notation of (2.1) below). Thus the exceptions occur only for simple sequences (i.e., when the companion polynomial has only simple roots), in this case the polynomial-exponential equations (2.2), (2.3) arising in Section 2 reduce to purely exponential equations. In the exceptions for Theorem 1 the roots $\alpha_{i}$ are algebraic (with respect to $a / c, b / c$ ).

An equation similar to (1.1) (with two terms instead of three) has been investigated by Schlickewei and Schmidt [SS2]. Related (linear) equations were studied by them in [SS1]. A basic tool are the results of Laurent ([L1], [L2]) on solutions of polynomial-exponential equations, and he already gave some applications to linear recurrence sequences (Laurent's result relies on the subspace theorem, see also [SS3], [A] and [ESS]). In the binary counterpart of (1.1), $a u_{n}^{l}=b u_{m}^{k}$, it turned out in [SS2] that (for non-degenerate sequences $\left(u_{n}\right)$ ) there are only "obvious" exceptions in which the equation has infinitely many solutions $(n, m)$. In the other case, quantitative estimates (with explicit bounds) for the number of solutions were given. In [SS2], Laurent's method led to a combinatorial problem on partitions of certain index sets. This was solved comparatively easily by an elegant argument and it turned out that the groups $\mathcal{H}(\mathcal{P})$ (see Section 4 below) are trivial for se- 
quences of rank $r>1$ and partitions $\mathcal{P}$ without singletons (also in most of the examples of [SS1] and [L1], [L2] a similar behaviour occurred).

In our case, for equation (1.2) one can use quite similar arguments, but at the end two partitions remain for which $\mathcal{H}(\mathcal{P})$ is non-trivial and they give rise to the exceptions in Theorem 2. For the ternary equation (1.1), we restrict to sequences of rank $r \geq 2$ (for $r=1$ there are still more new phenomena). The corresponding combinatorial question turns out to be somewhat more delicate. If $g(=\operatorname{rank}$ of $\mathcal{G})$ is at least 2, then (Proposition, Section 6) it is still true that $\mathcal{H}(\mathcal{P})$ is trivial for partitions without singletons (an exposition of this result is also given in $[\mathrm{G}]$; moreover, quantitative estimates for the number of solutions of (1.1) in this case are given there, [G, Th. 3.2, p. 13]). For $g=1$, there are quite a number of cases where $\mathcal{H}(\mathcal{P})$ is non-trivial, but further investigations show that nevertheless most of them contribute only finitely many solutions. At the end, four partitions remain that give rise to the exceptions of Theorem 1. Of course, the cases where (1.2) has infinitely many solutions induce exceptions for (1.1) (for appropriate sequences $\left(u_{n}\right)$ ) and in this way two of these partitions can be derived from those appearing in the study of (1.2). But the other two partitions (and the corresponding infinite families of solutions of (1.1)) are not derived from (1.2).

2. Polynomial-exponential equations. The basic approach in the proof of the two theorems is the same as in [SS2], [SS1]. We use the representation

$$
u_{n}=\sum_{i=1}^{r} f_{i}(n) \alpha_{i}^{n}
$$

(with non-zero complex polynomials $f_{i}$ ) of the given sequence $\left(u_{n}\right)$. Substituting (2.1) into equation (1.1) and expanding the powers leads as in [SS2, p. 1046] to an equation

$$
\begin{aligned}
\sum_{1 \leq i_{1} \leq \cdots \leq i_{k} \leq r} F_{i_{1} \ldots i_{k}}(h) \alpha_{i_{1}}^{h} \cdots & \alpha_{i_{k}}^{h}+\sum_{1 \leq i_{1} \leq \cdots \leq i_{l} \leq r} G_{i_{1} \ldots i_{l}}(n) \alpha_{i_{1}}^{n} \cdots \alpha_{i_{l}}^{n} \\
& +\sum_{1 \leq i_{1} \leq \cdots \leq i_{m} \leq r} H_{i_{1} \ldots i_{m}}(q) \alpha_{i_{1}}^{q} \cdots \alpha_{i_{m}}^{q}=0
\end{aligned}
$$

(see [G, p. 15] for more details) and this can be written as a polynomialexponential equation

$$
\sum_{\mathbf{i} \in A} p_{\mathbf{i}}(\mathbf{n}) \boldsymbol{\alpha}_{\mathbf{i}}^{\mathbf{n}}=0
$$

(with the usual notational conventions for exponents, see [SS2, p. 1045]). More explicitly, put $A_{k}=\left\{\mathbf{i}=\left(i_{1}, \ldots, i_{k}\right) \in \mathbb{N}^{k}: 1 \leq i_{1} \leq \cdots \leq i_{k} \leq r\right\}$ and similarly $A_{l}, A_{m}$ (recall that $k, l, m$ are different). Then $A=A_{k} \cup A_{l} \cup A_{m}$, 


$$
\begin{aligned}
& \mathbf{n}=(h, n, q) \in \mathbb{Z}^{3}, \\
& \boldsymbol{\alpha}_{\mathbf{i}}=\left\{\begin{array}{l}
\left(\alpha_{i_{1}} \cdots \alpha_{i_{k}}, 1,1\right), \\
\left(1, \alpha_{i_{1}} \cdots \alpha_{i_{l}}, 1\right), \\
\left(1,1, \alpha_{i_{1}} \cdots \alpha_{i_{m}}\right),
\end{array} \quad p_{\mathbf{i}}(\mathbf{n})= \begin{cases}F_{i_{1} \ldots i_{k}}(h) & \text { for } \mathbf{i} \in A_{k}, \\
G_{i_{1} \ldots i_{l}}(n) & \text { for } \mathbf{i} \in A_{l}, \\
H_{i_{1} \ldots i_{m}}(q) & \text { for } \mathbf{i} \in A_{m} .\end{cases} \right.
\end{aligned}
$$

Similarly, equation (1.2) leads to

$$
\sum_{\mathbf{i} \in A^{*}} q_{\mathbf{i}}(\mathbf{m}) \boldsymbol{\beta}_{\mathbf{i}}^{\mathbf{m}}=0
$$

with

$$
\mathbf{m}=(n, q) \in \mathbb{Z}^{2}, \quad A^{*}=A_{l} \cup A_{m} \cup\{*\}, \quad \boldsymbol{\beta}_{*}=(1,1), \quad q_{*}=-1 .
$$

(* stands for an additional index not belonging to $A_{l} \cup A_{m}$ and designating the constant term of the equation.)

Now one can use the results of Laurent [L2] on polynomial-exponential equations (an application of the subspace theorem). One has to study partitions $\mathcal{P}$ of $A$ and associated groups $\mathcal{H}(\mathcal{P})$ (see below for their definitions, and the beginning of Section 11). Partitions $\mathcal{P}$ for which $\mathcal{H}(\mathcal{P})$ is trivial contribute at most finitely many elements to the set of solutions of the equation (see e.g. [SS2, Th. A]).

The proof of the two theorems will be organized as follows: first (Section 3), we will handle the case of partitions $\mathcal{P}$ containing singletons. Then, for the case of partitions $\mathcal{P}$ without singletons, we will (Section 5) investigate consequences of the non-triviality of $\mathcal{H}(\mathcal{P})$ to the structure of $\mathcal{P}$. This leads to some combinatorial questions. In the case of Theorem 1 and $g \geq 2$, we will show in Section 6 that $\mathcal{H}(\mathcal{P})$ is trivial for partitions $\mathcal{P}$ without singletons (see also [G, Prop. 4.1]). Similarly for Theorem 2, where $\mathcal{H}(\mathcal{P})$ is trivial except for two cases for $\mathcal{P}$ leading to the exceptions (Section 7 ). For equation (1.1) (Theorem 1) with $g=1$, it can happen that $\mathcal{H}(\mathcal{P})$ is non-trivial (see the Remarks at the end of Section 10, containing a complete description of these partitions for $r=2,3$ ). But (as in [SS1]) the information that certain sets have to belong to $\mathcal{P}$ (Section 10) gives additional equations for the corresponding solutions (Section 11). Hence, the final step (Section 13) is the analysis of some of these additional equations. For partitions with non-trivial $\mathcal{H}(\mathcal{P})$ the corresponding solutions lead to polynomial identities (Section 12, (12.1)) and a large portion of these identities can be handled using the method of Mason ([M1, Lemma 2, p. 14]; this is a special case of the $a b c$-inequality in function fields which was used in [BMZ] to derive bounds for 0-multiplicities of linear recurrence sequences). By this approach (and there are also some limiting cases with small exponents to be discussed separately), we will be able to show that even if $\mathcal{H}(\mathcal{P})$ is non-trivial, only finitely many solutions exist (apart from the exceptions). 
3. Partitions with singletons. This means that we study first solutions $\mathbf{n}=(h, n, q)$ of (1.1) for which one term $p_{\mathbf{i}}(\mathbf{n})$ of the sum (2.2) is zero. Changing the order of the terms of (1.1) if necessary, we may assume that $\mathbf{i}=\left(i_{1}, \ldots, i_{k}\right) \in A_{k}$. By explicit computation (see [G, p. 15]), one can easily show that $F_{i_{1} \ldots i_{k}}$ is a non-zero multiple of the polynomial $\prod_{j=1}^{k} f_{i_{j}}$. There are only finitely many integers $h$ for which $\prod_{j=1}^{k} f_{i_{j}}(h)=0$. If $h_{0}$ is one of them and a solution $(h, n, q)$ of (1.1) satisfies $h=h_{0}$, this means that

$$
b u_{n}^{l}+c u_{q}^{m}=-a u_{h_{0}}^{k} .
$$

If $u_{h_{0}}=0$, then $(n, q)$ satisfy an equation of the type studied in [SS2], hence this gives only finitely many solutions. If $u_{h_{0}} \neq 0$ the resulting equation for $(n, q)$ is equivalent to (1.2), hence this reduces to Theorem 2. Thus (as soon as Theorem 2 has been shown), we know that the only instances where infinitely many solutions of (1.1) with $h=h_{0}$ can arise are the exceptional cases for Theorem 2. But by the proof of Theorem 2 in Section 7, this implies that the polynomials $f_{i}$ are constant, which is impossible for partitions with singletons.

In the case of partitions for (1.2) with singletons, the procedure is similar. One variable gets fixed, say $q=q_{0}$ (finitely many possibilities), and then a solution of (1.2) satisfies an equation $u_{n}=c^{\prime}$ (with finitely many possibilities for $c^{\prime}$ ). By the Skolem-Mahler-Lech Theorem, this has only finitely many solutions for a non-degenerate sequence with $r \geq 1$ (see [ST, Cor. C.1], the argument there holds for arbitrary $a$-multiplicities; see also [SS1, p. 229]).

Remark. For degenerate sequences (e.g., if $\alpha_{2 i}=-\alpha_{2 i-1}$ for $i \leq r / 2$ ), it can happen that $u_{n}=0$ for infinitely many $n$, and then equation (1.1) will clearly have infinitely many solutions. Similarly for (1.2).

4. The groups $\mathcal{H}(\mathcal{P})$. Given a partition $\mathcal{P}$ of the set $A$, the group $\mathcal{H}(\mathcal{P})$ consists of all $\mathbf{n} \in \mathbb{Z}^{3}$ such that $\boldsymbol{\alpha}_{\mathbf{i}}^{\mathbf{n}}$ is constant for $\mathbf{i} \in I$, whenever $I \in \mathcal{P}$ ([L2, p. 26]). Again we follow the procedure of [SS2, Sec. 5].

Let $\mathcal{T}$ be the torsion subgroup of $\mathcal{G}$. Then $\mathcal{G} / \mathcal{T} \cong \mathbb{Z}^{g}$, i.e., we have a surjective homomorphism $\psi: \mathcal{G} \rightarrow \mathbb{Z}^{g}$. Put $v_{i}=\psi\left(\alpha_{i}\right)(i=1, \ldots, r)$. For $\mathbf{i}=\left(i_{1}, \ldots, i_{k}\right) \in A_{k}$ put

$$
\sigma(\mathbf{i})=v_{i_{1}}+\cdots+v_{i_{k}}
$$

similarly for $\mathbf{i} \in A_{l} \cup A_{m}$. Moreover, $\psi$ induces a homomorphism $\mathcal{G}^{3} \rightarrow \mathbb{Z}^{g}$ (again denoted by $\psi$ ) by putting $\psi(\alpha, \beta, \gamma)=\psi(\alpha)+\psi(\beta)+\psi(\gamma)$. Then $\psi\left(\boldsymbol{\alpha}_{\mathbf{i}}\right)=\sigma(\mathbf{i})$ for all $\mathbf{i} \in A$. For a fixed $\mathbf{n}=(K, L, M) \in \mathbb{Z}^{3}$, put

$$
\bar{\sigma}(\mathbf{i})= \begin{cases}K \sigma(\mathbf{i}) & \text { for } \mathbf{i} \in A_{k}, \\ L \sigma(\mathbf{i}) & \text { for } \mathbf{i} \in A_{l}, \\ M \sigma(\mathbf{i}) & \text { for } \mathbf{i} \in A_{m} .\end{cases}
$$

Then $\psi\left(\boldsymbol{\alpha}_{\mathbf{i}}^{\mathbf{n}}\right)=\bar{\sigma}(\mathbf{i})$. 
Conversely, given $(K, L, M) \in \mathbb{Z}^{3}$ and arbitrary vectors $v_{1}, \ldots, v_{r} \in \mathbb{R}^{g}$, we can define functions $\sigma$ and $\bar{\sigma}$ by (4.1) and (4.2). Those sets of the form $\bar{\sigma}^{-1}(v)$ for $v \in \mathbb{R}^{g}$ that are non-empty constitute a partition $\mathcal{P}_{\bar{\sigma}}$ of $A$. It is easy to see that if $v_{i}=\psi\left(\alpha_{i}\right)$ as above, then some multiple of $(K, L, M)$ (depending on the roots of unity in $\mathcal{T}$ ) will belong to $\mathcal{H}\left(\mathcal{P}_{\bar{\sigma}}\right)$.

On the other hand, if we start with some $(K, L, M) \in \mathcal{H}(\mathcal{P})$, then $\mathcal{P}_{\bar{\sigma}}$ is coarser than $\mathcal{P}$ (or possibly equal). Hence, if $\mathcal{P}$ contains no singletons (i.e., one-element sets), then the same will be true for $\mathcal{P}_{\bar{\sigma}}$. For our purpose (see also the beginning of Section 11), it will be enough to study partitions $\mathcal{P}_{\bar{\sigma}}$. Our first target (Sections 5 and 10) will be to derive consequences of the assumption on $\mathcal{P}_{\bar{\sigma}}$ to contain no singletons, i.e., what happens if $\bar{\sigma}$ takes every value at least twice.

For equation (1.2), the procedure is similar. We have to study partitions of $A^{*}$. On $A_{l} \cup A_{m}, \sigma$ and $\bar{\sigma}$ are defined as in (4.1), (4.2) and we put $\sigma(*)=\bar{\sigma}(*)=0$.

5. Properties of $\bar{\sigma}$. The foundation is laid by the case $g=1$. Hence, we now assume that $r \geq 2$ and that $v_{1}<\cdots<v_{r}$ are real numbers. We fix a non-zero triple $(K, L, M) \in \mathbb{Z}^{3}$ (or $\mathbb{R}^{3}$ ). As in [SS2, Proof of the Proposition], we study the values of $\bar{\sigma}$ close to the minimum resp. maximum under the assumption that $\mathcal{P}_{\bar{\sigma}}$ has no singletons. This will lead to a classification into cases A, B with subcases Baa, Bab, Bba, Bbb, Bbc.

For notational distinction, we often include the length of an ordered tuple as an index, e.g. $(1, \ldots, 1)_{k}$ denotes a $k$-tuple. Remember also that $A$ contains only non-decreasing tuples, hence the notation $(\ldots, 1,2)_{k}$ determines the initial segment uniquely. For $k=1,(\ldots, 1,2)_{k}$ is to be read as (2) and $(r-1, r, \ldots)_{k}$ stands for $(r-1)$.

5.1. If $K>0$, then $\bar{\sigma}(1, \ldots, 1)_{k}$ gives the smallest value of $\bar{\sigma}$ on $A_{k}$, $\bar{\sigma}(\ldots, 1,2)_{k}$ is second smallest, $\bar{\sigma}(r, \ldots, r)_{k}$ the largest and $\bar{\sigma}(r-1, r, \ldots)_{k}$ the second largest. These four values are taken only once on $A_{k}$.

5.2. If $K, L, M \geq 0, \mathcal{P}_{\bar{\sigma}}$ has no singletons and $v_{1}<0$, then there is a (uniquely determined) reordering of $(k, l, m)$ (and $(K, L, M)$ ) such that the two smallest values of $\bar{\sigma}$ on $A$ are given by $\bar{\sigma}(1, \ldots, 1)_{l}=\bar{\sigma}(1, \ldots, 1)_{m}$ (the minimum) and $\bar{\sigma}(\ldots, 1,2)_{l}=\bar{\sigma}(1, \ldots, 1)_{k}$. (For $K>0$, these are the only elements of $A$ giving these values.) This results in the following relations (CASE A):

$$
\begin{gathered}
0 \leq K k<L l=M m, \quad L<M, \\
K k v_{1}=L(l-1) v_{1}+L v_{2} .
\end{gathered}
$$

Proof. Since $(K, L, M) \neq(0,0,0)$, we may assume that $L>0$. By Property 5.1 , we may assume that $\bar{\sigma}(1, \ldots, 1)_{l}$ is the minimum of $\bar{\sigma}$ on $A$ (observe 
that $\left.\bar{\sigma}(1, \ldots, 1)_{l}<0\right)$. Since this value is attained only once on $A_{l}$, we may assume that $\bar{\sigma}(1, \ldots, 1)_{l}=\bar{\sigma}(1, \ldots, 1)_{m}$, giving $L l=M m$. By assumption $l \neq m$, hence possibly after reordering, we get $L<M$.

Now assume that $K k=L l=M m$, i.e., $\bar{\sigma}(1, \ldots, 1)_{k}=\bar{\sigma}(1, \ldots, 1)_{l}$. Then we may also assume that $L<K$. The distances of $\bar{\sigma}(1, \ldots, 1)_{l}$ to the next smallest values on $A_{l}, A_{k}, A_{m}$ are $L\left(v_{2}-v_{1}\right), K\left(v_{2}-v_{1}\right), M\left(v_{2}-v_{1}\right)$, respectively. Hence $(\ldots, 1,2)_{l}$ would give a singleton for $\mathcal{P}_{\bar{\sigma}}$. Thus we get $K k<L l$ (since $\left.\bar{\sigma}(1, \ldots, 1)_{k} \geq \bar{\sigma}(1, \ldots, 1)_{l}\right)$ and, as above, to avoid singletons, we must have $\bar{\sigma}(\ldots, 1,2)_{l}=\bar{\sigma}(1, \ldots, 1)_{k}$, i.e., $L(l-1) v_{1}+L v_{2}=K k v_{1}$.

In the case of different signs among $K, L, M$ there are more possibilities (see also [G, Prop. 4.3, Teil I of the proof] for a more detailed exposition of the classification). Since $\bar{\sigma}$ and $-\bar{\sigma}$ define the same partition of $A$, we can assume (possibly after reordering) that $K<0, L, M \geq 0$ (CASE B).

5.3. (CASE Ba) If $K<0, L, M \geq 0, L l>M m$ and $\mathcal{P}_{\bar{\sigma}}$ has no singletons, then the smallest value of $\bar{\sigma}$ on $A$ is given by $\bar{\sigma}(1, \ldots, 1)_{l}=$ $\bar{\sigma}(r, \ldots, r)_{k}$ and the largest by $\bar{\sigma}(r, \ldots, r)_{l}=\bar{\sigma}(1, \ldots, 1)_{k}$. This results in the following relations:

$$
v_{r}=-v_{1}, \quad 0 \leq M m<L l=-K k, \quad L \neq-K .
$$

Proof. First assume that $v_{1}<0$. Since $K<0$, the indices in the description of the maximal and minimal values of $\bar{\sigma}\left(A_{k}\right)$ in Property 5.1 have to be reflected. The assumption $L l>M m$ implies $\bar{\sigma}(1, \ldots, 1)_{l}<\bar{\sigma}(1, \ldots, 1)_{m}$, hence arguing as before, we conclude that $\bar{\sigma}(1, \ldots, 1)_{l}=\bar{\sigma}(r, \ldots, r)_{k}$ gives the minimum of $\bar{\sigma}$. In particular, $v_{r}>0$. Then the same argument, used for the maximum, gives $\bar{\sigma}(r, \ldots, r)_{l}=\bar{\sigma}(1, \ldots, 1)_{k}$. From $L l v_{1}=K k v_{r}$, $L l v_{r}=K k v_{1}$, it follows that $v_{r}=-v_{1}, L l=-K k$ and then, $l \neq k$ implies $L \neq-K$.

If $v_{1} \geq 0$, then $v_{r}>0$ and the same argument as above, used for the maximum, would give $v_{1}<0$, a contradiction.

5.4. Under the assumptions of 5.3 (Case $\mathrm{Ba})$, we have $v_{r-1}=-v_{2}$ and concerning the second smallest (largest) values of $\bar{\sigma}$ there are the following two possibilities:

CASE Baa: $L<-K, L(l-1) v_{1}+L v_{2}=M m v_{1}$.

CAse Bab: $L>-K, K(k-1) v_{1}+K v_{2}=M m v_{r}$.

(For $M>0$ we get again all elements of $A$ that are mapped to the smallest value, second smallest value, etc.)

Proof. We may assume that $v_{2}-v_{1} \leq v_{r}-v_{r-1}$, otherwise we consider the reverted sequence $v_{i}^{*}=-v_{r+1-i}$ (which produces a partition arising from $\mathcal{P}_{\bar{\sigma}}$ by a corresponding transformation). As in the proof of 5.2 , we consider the distances of $\bar{\sigma}(1, \ldots, 1)_{l}$ to the next smallest values on $A_{k}$ and $A_{l}$. Then 
$L<-K$ implies that $\bar{\sigma}(\ldots, 1,2)_{l}=\bar{\sigma}(1, \ldots, 1)_{m}$ is the second smallest value of $\bar{\sigma}$, i.e. $L(l-1) v_{1}+L v_{2}=M m v_{1}$. Then $L l v_{r}-M m v_{r}=-(L l-M m) v_{1}=$ $L\left(v_{2}-v_{1}\right)<-K\left(v_{2}-v_{1}\right)$ and it follows that $\bar{\sigma}(r-1, r, \ldots)_{l}=\bar{\sigma}(r, \ldots, r)_{m}$ gives the second largest value, i.e. $L(l-1) v_{r}+L v_{r-1}=M m v_{r}$, and from $v_{r}=-v_{1}$ (see 5.3) we get $v_{r-1}=-v_{2}$.

Similarly, if $L>-K$ holds, one shows that $\bar{\sigma}(r-1, r, \ldots)_{k}=\bar{\sigma}(1, \ldots, 1)_{m}$ and $K(k-1) v_{r}+K v_{r-1}=-M m v_{1}, L l v_{r}-M m v_{r}=-K\left(v_{r}-v_{r-1}\right)<$ $L\left(v_{r}-v_{r-1}\right)$, which results in $\bar{\sigma}(\ldots, 1,2)_{k}=\bar{\sigma}(r, \ldots, r)_{m}$.

5.5. (CASE $\mathrm{Bb)}$ If $K<0, L, M \geq 0, L l=M m, L<M$ and $\mathcal{P}_{\bar{\sigma}}$ has no singletons, then the smallest value of $\bar{\sigma}$ on $A$ is given by $\bar{\sigma}(1, \ldots, 1)_{l}=$ $\bar{\sigma}(1, \ldots, 1)_{m}$ and the largest by $\bar{\sigma}(r, \ldots, r)_{l}=\bar{\sigma}(r, \ldots, r)_{m}$, in particular $-K k<L l$. (But here, one cannot exclude that one of these values is attained also on $A_{k}$, see 5.6.)

Proof. As in 5.3. If $\bar{\sigma}(1, \ldots, 1)_{l}>\bar{\sigma}(r, \ldots, r)_{k}$, then $(r, \ldots, r)_{k}$ would give a singleton.

5.6. Under the assumptions of 5.5 (Case $\mathrm{Bb}$ ), we have the following three possibilities, resulting from the second smallest (largest) values of $\bar{\sigma}$ :

CASE Bba: $\quad L(l-1) v_{1}+L v_{2}=K k v_{r}, L(l-1) v_{r}+L v_{r-1}=K k v_{1}$.

CASE Bbb: $L l v_{1}=K k v_{r}, L(l-1) v_{r}+L v_{r-1}=K k v_{1}$.

CASE Bbc: $L(l-1) v_{1}+L v_{2}=K k v_{r}, L l v_{r}=K k v_{1}$.

Proof. The assumption $L<M$ gives $\bar{\sigma}(\ldots, 1,2)_{l}<\bar{\sigma}(\ldots, 1,2)_{m}$. Hence, if $\bar{\sigma}(1, \ldots, 1)_{l}<\bar{\sigma}(r, \ldots, r)_{k}$ (i.e., $\left.L l v_{1}<K k v_{r}\right)$, then $\bar{\sigma}(\ldots, 1,2)_{l}=$ $\bar{\sigma}(r, \ldots, r)_{k}$ is the second smallest value of $\bar{\sigma}$ on $A$, i.e., $L(l-1) v_{1}+L v_{2}=$ $K k v_{r}$. Similarly, $\bar{\sigma}(r, \ldots, r)_{l}>\bar{\sigma}(1, \ldots, 1)_{k}$ implies that $\bar{\sigma}(r-1, r, \ldots)_{l}=$ $\bar{\sigma}(1, \ldots, 1)_{k}$, i.e., $L(l-1) v_{r}+L v_{r-1}=K k v_{1}$. Thus, it remains to exclude the possibility that $L l v_{1}=K k v_{r}$ and $L l v_{r}=K k v_{1}$. As in 5.3 this would imply $v_{r}=-v_{1}, L l=-K k$ and the second smallest (resp. largest) value would be $\bar{\sigma}(\ldots, 1,2)_{l}=\bar{\sigma}(r-1, r, \ldots)_{k}\left(\right.$ resp. $\left.\bar{\sigma}(r-1, r, \ldots)_{l}=\bar{\sigma}(\ldots, 1,2)_{k}\right)$. Considering the distance to the minimum (resp. maximum), this would imply $L\left(v_{2}-v_{1}\right)=K\left(v_{r}-v_{r-1}\right)$ (resp. $\left.L\left(v_{r-1}-v_{r}\right)=K\left(v_{1}-v_{2}\right)\right)$, which gives $L=-K$, contradicting $l \neq k$.

REMARK. Reflecting the sequence $\left(v_{i}\right)$ (i.e., replacing it by $v_{i}^{*}=-v_{r+1-i}$ ) transfers Case Bbc to Bbb and conversely. Similarly, for $M=0$, multiplying $(K, L, M)$ by -1 and reordering transfers Case Baa to Bab and conversely.

Up to reordering the sequence $\left(v_{i}\right)$, multiplying $(K, L, M)$ by -1 and/or changing the order of $k, l, m$, the preceding properties classify all partitions $\mathcal{P}_{\bar{\sigma}}$ without singletons for $g=1, r \geq 2$. (Apart from the two overlappings described just before, the various cases are distinct.) This can be summarized as follows (the "main cases" A, Baa, Bab, Bba): the two larger ones of the 
three values $|K k|,|L l|,|M m|$ coincide and they determine the maximum and the minimum of $\bar{\sigma}$. The third one determines the second smallest (largest) value. The different cases represent the various distributions of signs among $K, L, M$ in relation to the "third" value. The only exceptions are Cases Bbb, $\mathrm{Bbc}$, where one of the two extremal values of $\bar{\sigma}(A)$ is taken three times (this happens also in Case A for $v_{r}=0$ ). We will see later (Property 10.11) that (at least for $K, L, M \in \mathbb{Z}$ ) these cases occur only rarely, i.e., only for $r=2$ and with further restrictions on $k, l, m$. Nevertheless, they contribute to the exceptions of Theorem 1 described in Section 9.

\section{The case $g \geq 2$}

Proposition. Take $(K, L, M) \in \mathbb{R}^{3} \backslash\{\mathbf{0}\}$, consider distinct elements $v_{1}, \ldots, v_{r} \in \mathbb{R}^{g}$ and define $\bar{\sigma}: A \rightarrow \mathbb{R}^{g}$ by (4.2). Assume that $v_{1}, \ldots, v_{r}$ are not contained in a one-dimensional subspace of $\mathbb{R}^{g}$. Then there exists $v \in \mathbb{R}^{g}$ such that $\bar{\sigma}^{-1}(v)$ consists of a single point (i.e., the partition $\mathcal{P}_{\bar{\sigma}}$ of A contains a singleton).

Proof. (a) We assume that $\mathcal{P}_{\bar{\sigma}}$ has no singletons. Following the method of [SS2, Proof of the Proposition], we consider linear mappings $q: \mathbb{R}^{g} \rightarrow \mathbb{R}$. Observe that if $q$ is any linear mapping, then $q \circ \bar{\sigma}$ satisfies again (4.2), when using $q\left(v_{1}\right), \ldots, q\left(v_{r}\right)$ in place of $v_{1}, \ldots, v_{r}$. The partition $\mathcal{P}_{q \circ \bar{\sigma}}$ is coarser than $\mathcal{P}_{\bar{\sigma}}$ (or possibly equal), hence $\mathcal{P}_{q \circ \bar{\sigma}}$ will always contain no singletons. We call a linear mapping $q: \mathbb{R}^{g} \rightarrow \mathbb{R}$ admissible if the values $q\left(v_{1}\right), \ldots, q\left(v_{r}\right)$ are pairwise different. As observed in [SS2], the set of admissible mappings $q$ consists of the complement of finitely many hyperplanes in the dual of $\mathbb{R}^{g}$.

Now let $q$ be an admissible mapping. Possibly after reordering, we may assume that $q\left(v_{1}\right)<\cdots<q\left(v_{r}\right)$ and (reflecting the sequence $v_{1}, \ldots, v_{r}$ if necessary) that $q\left(v_{1}\right)<0$ (clearly our assumption implies that $r \geq 2$ ). We can apply the properties derived in Section 5 to $q \circ \bar{\sigma}$ and consider the cases from there.

(b) Assume that we have Case A, in particular, $K, L, M \geq 0$. Then by Property $5.2,\left\{(\ldots, 1,2)_{l},(1, \ldots, 1)_{k}\right\} \in \mathcal{P}_{q \circ \bar{\sigma}}$ and $L>0$ by (5.1). Passing from $\mathcal{P}_{q \circ \bar{\sigma}}$ to the finer partition $\mathcal{P}_{\bar{\sigma}}$, two-element or three-element sets cannot be split (since this would generate singletons). Hence $\bar{\sigma}(\ldots, 1,2)_{l}=$ $\bar{\sigma}(1, \ldots, 1)_{k}$ and it follows that $K k v_{1}=L(l-1) v_{1}+L v_{2}$ (alternatively, it would be no problem to require that $q$ is injective on the finite set $\bar{\sigma}(A)$ ). Thus, $v_{1}, v_{2}$ are linearly dependent.

If $r=2$, this gives already a contradiction, hence we can assume that $r>2$. Observe that by the equation above, $v_{2}$ is determined uniquely by $v_{1}$. Hence, if $q^{\prime}$ is any other admissible mapping having $q^{\prime}\left(v_{1}\right)$ as minimal value, then $q^{\prime}\left(v_{2}\right)$ should give the second smallest value. 
Since $v_{1}, \ldots, v_{r}$ do not lie in a one-dimensional subspace, there exists $w \perp v_{1}-v_{2}$ such that $\left(v_{i} \mid w\right)<0$ for some $i>2((\cdot \mid \cdot)$ denotes the standard inner product). For $t \in \mathbb{R}$, put $q_{t}(v)=q(v)+t(v \mid w)$. Choose $i>2$ such that $c_{0}=\left(v_{i} \mid w\right)$ is minimal and put $t_{0}=q\left(v_{2}-v_{i}\right) / c_{0}$. Then $t_{0}>0$, and $\widetilde{q}=q_{t_{0}}$ satisfies $q\left(v_{2}\right)=\widetilde{q}\left(v_{2}\right)=\widetilde{q}\left(v_{i}\right) \leq \widetilde{q}\left(v_{j}\right)$ for all $j \geq 2$ and $q\left(v_{1}\right)=$ $\widetilde{q}\left(v_{1}\right)<\widetilde{q}\left(v_{2}\right)$ (observe that $\left(v_{1} \mid w\right)=\left(v_{2} \mid w\right)=0$ by linear dependence). Now, $q_{t}$ is admissible except for finitely many $t$ (it is easy to see that if $q_{t}$ is not admissible for infinitely many $t$, then it would be non-admissible for all $t$, contradicting $q_{0}=q$ ). Choose $t>t_{0}$ such that $q_{t}$ is admissible and $t-t_{0}$ small. Then $q_{t}\left(v_{1}\right)$ is still the minimum, but $q_{t}\left(v_{i}\right)<q_{t}\left(v_{2}\right)$ and this gives a contradiction.

(c) The same argument works in Case Ba (using Property 5.4). Similarly, one can treat Case Bbc (note that $\left\{(1, \ldots, 1)_{k},(r, \ldots, r)_{l},(r, \ldots, r)_{m}\right\} \in$ $\mathcal{P}_{q \circ \bar{\sigma}}$ implies $K k v_{1}=L l v_{r}$ and then Case Bbc has to occur for any other admissible mapping $q^{\prime}$ with the property that $q^{\prime}\left(v_{1}\right)$ is minimal). Case Bbb can be transferred to Bbc by reflecting the sequence $\left(v_{i}\right)$.

(d) Case Bba causes more difficulties. There are various ways to handle it (see the Remark below); we present here a geometric argument. Put $B=\left\{v_{1}, \ldots, v_{r}\right\}$ and let $C$ be the convex hull of $B$. As above, let $q$ be an admissible mapping. We may assume (see (c)) that Case Bba occurs for any such mapping. We take the ordering so that $q\left(v_{1}\right)<\cdots<q\left(v_{r}\right)$. Then the argument as in (b) and Property 5.6 give $K k v_{r}=L(l-1) v_{1}+L v_{2}$. This can be written as $v_{r}=c\left(\lambda v_{1}+(1-\lambda) v_{2}\right)$, with $c=L l / K k<0$ and $\lambda=1-1 / l$. Observe that $L<M$ implies $l \geq 2$, hence $0<\lambda<1$. Similarly, we have $v_{1}=c\left(\lambda v_{r}+(1-\lambda) v_{r-1}\right)$.

For $r=2$ this implies that $v_{1}, v_{2}$ are linearly dependent, hence we can assume $r>2$. Note that by the equations, $v_{r-1}$ and $v_{r}$ are determined uniquely by $v_{1}, v_{2}$. Hence, if $q^{\prime}$ is any other admissible mapping such that $q^{\prime}\left(v_{1}\right)<q^{\prime}\left(v_{2}\right)<q^{\prime}\left(v_{j}\right)$ for all $j>2$, then $q^{\prime}\left(v_{r}\right)>q^{\prime}\left(v_{r-1}\right)>q^{\prime}\left(v_{j}\right)$ for all $j<r-1$. Similarly, if $q^{\prime}$ is admissible, $q^{\prime}\left(v_{1}\right)$ is minimal, $q^{\prime}\left(v_{r}\right)$ is maximal, then $q^{\prime}\left(v_{2}\right)$ has to be the second smallest value.

Now define $q_{t}, t_{0}$ and $\widetilde{q}$ as in (b). We have $\widetilde{q}\left(v_{2}-v_{1}\right)=q\left(v_{2}-v_{1}\right)>0$. In the limit for $t \rightarrow t_{0}$ - with $q_{t}$ admissible, we see from the observation above that $\widetilde{q}\left(v_{r}\right) \geq \widetilde{q}\left(v_{r-1}\right) \geq \widetilde{q}\left(v_{j}\right)$ for $j<r-1$ and $\widetilde{q}\left(v_{1}\right)<\widetilde{q}\left(v_{2}\right) \leq \widetilde{q}\left(v_{j}\right)$ for $j>2$. If it happened that $\widetilde{q}\left(v_{r}\right)>\widetilde{q}\left(v_{r-1}\right)$, then for $t>t_{0}$ and $t-t_{0}$ small, $q_{t}\left(v_{r}\right)$ would still be the maximum and $q_{t}\left(v_{1}\right)$ the minimum. For $q_{t}$ admissible, this would imply $q_{t}\left(v_{2}\right)<q_{t}\left(v_{j}\right)$ for $j>2$, a contradiction to the choice of $t_{0}$. Hence we must have $\widetilde{q}\left(v_{r}\right)=\widetilde{q}\left(v_{r-1}\right)$.

In particular, if the dimension of the vector space generated by $B$ were greater than 2 , we could choose $w \perp v_{1}, v_{2}$. Then $\widetilde{q}\left(v_{r}\right)=q\left(v_{r}\right)>q\left(v_{r-1}\right)=$ $\widetilde{q}\left(v_{r-1}\right)$ and this is impossible. Hence, we can assume that $g=2$, i.e., $C \subseteq \mathbb{R}^{2}$. 


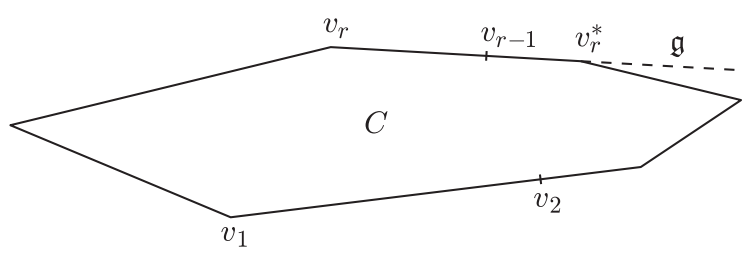

Fig. 1

Continuing the investigation of $q_{t}$, let $\mathfrak{g}=\left\{v \in \mathbb{R}^{2}: \widetilde{q}(v)=\widetilde{q}\left(v_{r}\right)\right\}$ be the line passing through $v_{r-1}, v_{r}$ (see Fig. 1). From $t_{0}>0, q\left(v_{r-1}\right)<q\left(v_{r}\right)$, we find that $\left(v_{r} \mid w\right)<\left(v_{r-1} \mid w\right)$. Let $v=v_{r}^{*}$ give the maximal value of $(v \mid w)$ on $B \cap \mathfrak{g}$ and $v_{r-1}^{*}$ the second largest. Since for $t>t_{0}$ with $t-t_{0}$ small, the largest and second largest values of $q_{t}$ on $B$ must be given by points of $\mathfrak{g}$, these are attained at $v_{r}^{*}$ and $v_{r-1}^{*}$ respectively. For $q_{t}$ admissible, this entails $v_{1}=c\left(\lambda v_{r}^{*}+(1-\lambda) v_{r-1}^{*}\right)$. Thus $v_{1} / c$ is a point of $\mathfrak{g}$ lying between $v_{r}, v_{r-1}$ and between $v_{r-1}^{*}, v_{r}^{*}$. It follows that $v_{r}^{*}=v_{r-1}, v_{r-1}^{*}=v_{r}, \lambda=1 / 2$. This also implies that $B \cap \mathfrak{g}=\left\{v_{r-1}, v_{r}\right\}$.

Since $v_{1}$ is the unique point where $q$ attains its minimum on $C$, it has to be a vertex (extreme point) of $C$. Similarly, $v_{r}$ and $v_{r-1}\left(=v_{r}^{*}\right)$ have to be vertices. Hence, $v_{2}$ (giving the second largest value of the admissible mapping $-q$ ) has to be a vertex as well. Let $v_{2}^{*} \in B$ give the second smallest value of $q_{t}$ for $t>t_{0}, t-t_{0}$ small. Then, as above, we get $\widetilde{q}\left(v_{2}^{*}\right)=\widetilde{q}\left(v_{2}\right)$, $v_{r-1}\left(=v_{r}^{*}\right)=\frac{c}{2}\left(v_{1}+v_{2}^{*}\right)$ and $v_{2}^{*}$ is a vertex (it is the adjacent vertex to $v_{1}$ lying opposite to $\left.v_{2}\right)$.

Since for any $\bar{v} \in B$ that is a vertex of $C$ we can find an admissible mapping $q^{\prime}$ such that $q^{\prime}(\bar{v})$ is minimal, the results obtained so far can be summarized as follows: All elements of $B$ lying on the boundary of $C$ are vertices. The interior of the triangle spanned by any three consecutive vertices of $C$ contains no elements of $B$.

Now, $\lambda=1 / 2=1-1 / l$ implies $l=2$, and $M>L$ gives $m<l$, thus $m=1, M=2 L, k \geq 3$ (i.e., concerning (1.1) we are left with the equation of Section 9). In particular, $\bar{\sigma}\left(A_{m}\right) \subseteq \bar{\sigma}\left(A_{l}\right)$. We have $\bar{\sigma}(r-1, r, \ldots)_{k}=$ $K(k-1) v_{r}+K v_{r-1}=L v$ with $v=v_{1}+(1-1 / k) v_{2}+(1 / k) v_{2}^{*}$. To avoid singletons, there must be some $\mathbf{i}=\left(i_{1}, i_{2}\right) \in A_{l}$ such that $\bar{\sigma}(\mathbf{i})=L v$, i.e., $\sigma(\mathbf{i})=v$. Since $\widetilde{q}(v)=\widetilde{q}\left(v_{1}\right)+\widetilde{q}\left(v_{2}\right)$ and $\widetilde{q}(\sigma(\mathbf{i})) \geq 2 \widetilde{q}\left(v_{2}\right)$ as soon as $i_{1}>1$, we must have $i_{1}=1$ and it follows that $v_{i_{2}}=(1-1 / k) v_{2}+(1 / k) v_{2}^{*}$ belongs to $B$.

Since $\widetilde{q}\left(v_{1}\right)=c \widetilde{q}\left(v_{r}\right)<0$, we may by scaling assume that $\widetilde{q}\left(v_{1}\right)=-1$. Let $v_{j} \in B$ be the adjacent vertex to $v_{2}$ opposite to $v_{1}$, and $v_{j}^{\prime}$ be the adjacent vertex to $v_{r}$ opposite to $v_{r-1}$. As above, we have $v_{j_{0}}:=(1-1 / k) v_{1}$ $+(1 / k) v_{j} \in B$. Using the equations $v_{j}^{\prime}=\frac{c}{2}\left(v_{j}+v_{2}\right), v_{2}=\frac{c}{2}\left(v_{j}^{\prime}+v_{r}\right)$, one gets

$$
\widetilde{q}\left(v_{j_{0}}-v_{1}\right)=\frac{4}{k c^{2}} \widetilde{q}\left(v_{2}-v_{1}\right),
$$


hence $4 /\left(k c^{2}\right) \geq 1$. On the other hand, $\widetilde{q}\left(v_{2}\right)=1-2 / c^{2}>\widetilde{q}\left(v_{1}\right)=-1$ implies $c<-1$ and it follows that $k=3$. The last step is to show that $\left(1, j_{0}\right) \in A_{l}$ gives a singleton for $\mathcal{P}_{\bar{\sigma}}$.

The line through $v_{j}^{\prime}, v_{r-1}$ intersects the line through $\mathbf{0}, v_{1}$ at $(2 / c-c) v_{1}$ (since $\left.v_{r-1}+\frac{c^{2}}{4}\left(v_{j}^{\prime}-v_{r-1}\right)=(2 / c-c) v_{1}\right)$. We apply the statement about $B$ made above to the triangle spanned by $v_{j}^{\prime}, v_{r}, v_{r-1}$ and the next one. This gives $\widetilde{q}\left(v_{i}\right) \leq c-2 / c$ for all $i<r-1$. It follows (recall that $c=\frac{2}{3} L / K$ ) that $K \widetilde{q}\left(v_{i}\right) \geq \frac{2}{3} L\left(1-2 / c^{2}\right)$. Hence, for $\mathbf{i}=\left(j_{1}, j_{2}, j_{3}\right) \in A_{k}$ with $j_{1}<r-1$, we get

$$
\widetilde{q}(\bar{\sigma}(\mathbf{i})) \geq \frac{2}{3} L\left(1-\frac{2}{c^{2}}-2 \frac{1}{c^{2}}\right),
$$

and consequently

$$
\widetilde{q}\left(\bar{\sigma}(\mathbf{i})-2 L v_{1}\right) \geq 2 L \frac{4}{3}\left(1-\frac{1}{c^{2}}\right) .
$$

On the other hand, one computes

$$
\widetilde{q}\left(\bar{\sigma}\left(1, j_{0}\right)-2 L v_{1}\right)=2 L \frac{4}{3} \frac{c^{2}-1}{c^{4}}<4 L\left(1-\frac{1}{c^{2}}\right)=2 L \widetilde{q}\left(v_{2}-v_{1}\right) .
$$

The first inequality shows that $\bar{\sigma}\left(1, j_{0}\right) \neq \bar{\sigma}\left(j_{1}, j_{2}, j_{3}\right)$ if $j_{1}<r-1$. But for $j_{1} \geq r-1$, we have $\widetilde{q}\left(\bar{\sigma}\left(j_{1}, j_{2}, j_{3}\right)\right)=2 L \widetilde{q}\left(v_{2}\right)$, which is excluded by the second inequality. This shows that $\bar{\sigma}\left(1, j_{0}\right) \notin \bar{\sigma}\left(A_{k}\right)$, and in a similar way one gets $\bar{\sigma}\left(1, j_{0}\right) \neq \bar{\sigma}\left(j_{1}, j_{2}\right)$ for $\left(j_{1}, j_{2}\right) \neq\left(1, j_{0}\right)$ (which also entails that $\left.\bar{\sigma}\left(1, j_{0}\right) \notin \bar{\sigma}\left(A_{m}\right)\right)$. This finishes the proof of the Proposition.

REMARK. An alternative to the last (computational) part of (d) would be the observation that the equations $v_{1}=\frac{c}{2}\left(v_{r-1}+v_{r}\right)$ etc. for the points of $B$ lying on the boundary of $C$ give an eigenvalue system for the components of the $v_{i}$. The right hand side defines a circulant matrix and for the eigenvalue one gets $2 / c=2 \cos \left(\pi j / r_{0}\right)$ where $r_{0}$ is the number of points of $B$ on the boundary, $1 \leq j<r_{0}, j \in \mathbb{N}(c= \pm 1$ has already been excluded). For $K, L, M \in \mathbb{Z}$ (which entails $c \in \mathbb{Q}$ ), this would imply that $r_{0}=3$, i.e., $C$ would be a triangle, and this case has been excluded before.

It will be shown later (Property 10.9) by more elaborate investigations that in Case Bba (at least for $K, L, M \in \mathbb{Z}, r>3$ ) one always has $v_{r}=-v_{1}$, and then the easy argument of (b) can be applied.

Another method of proof which is more in the spirit of Section 10 of our paper can be found in $[\mathrm{G}]$.

7. Proof of Theorem 2. Starting from (1.2), we now have to consider partitions $\mathcal{P}$ of $A^{*}$. If $\mathcal{P}$ has singletons, then we have shown in Section 3 that there are only finitely many solutions. Partitions without singletons can be handled as in [SS2, Proof of the Proposition]. Take $(L, M) \in \mathcal{H}(\mathcal{P}) \backslash\{\mathbf{0}\}$ 
and define $\sigma, \bar{\sigma}$ as explained at the end of Section 4. Assume first that $L, M \geq 0$. In addition, we can assume $v_{1}<0$, otherwise replace $v_{i}$ by $v_{i}^{*}=-v_{r+1-i}$. It follows as in [SS2] or in Property 5.2 that $L l=M m$. Then $v_{1} \neq 0$ implies $r \geq 2$ (otherwise, $\{*\}$ would be a singleton). Ordering so that $0<L<M$, it follows that $\bar{\sigma}(\ldots, 1,2)_{l}=\bar{\sigma}(*)=0$ and that $\bar{\sigma}(r-1, r, \ldots)_{l}=\bar{\sigma}(*)=0$. This implies $r=2, l=2, m=1, v_{2}=-v_{1}$ and we arrive at the partition $\mathcal{P}_{1}=\{\{(1,1),(1)\},\{(1,2), *\},\{(2,2),(2)\}\}$. It follows from [L2, Lemma 7] that $f_{1}$ and $f_{2}$ are constant (see also Consequence 11.1). We write $\alpha_{1}=\alpha$, then $\alpha_{2}=1 /(\zeta \alpha)$, where $\zeta$ is a root of unity and we have $u_{n}=a_{1} \alpha^{n}+a_{2}(\zeta \alpha)^{-n}$. We are looking for solutions of $b u_{n}^{2}+c u_{n}=1$. The equations corresponding to $\mathcal{P}_{1}$ (i.e., for $(n, q) \in \bar{S}\left(\mathcal{P}_{1}\right)$; compare the beginning of Section 11) are:

$$
b a_{1}^{2} \alpha^{2 n}+c a_{1} \alpha^{q}=0, \quad 2 b a_{1} a_{2} \zeta^{-n}=1, \quad b a_{2}^{2}(\zeta \alpha)^{-2 n}+c a_{2}(\zeta \alpha)^{-q}=0 .
$$

Putting $t=q-2 n$, we get by elementary computations $b=2 c^{2} \zeta^{-n-t}$, $a_{2}=a_{1} \zeta^{-t} \alpha^{-2 t}, a_{1}=-\alpha^{t} \zeta^{n+t} /(2 c)$. Writing $\eta=\zeta^{-n-t}$ gives the form stated in Theorem 2.

In the case $L>0>M$, a similar argument gives $r, l, m, v_{i}$ as above and $\mathcal{P}_{2}=\{\{(1,1),(2)\},\{(1,2), *\},\{(2,2),(1)\}\}$. If we write $u_{n}$ as above, the equations for $\mathcal{P}_{2}$ are

$$
b a_{1}^{2} \alpha^{2 n}+c a_{2}(\zeta \alpha)^{-q}=0, \quad 2 b a_{1} a_{2} \zeta^{-n}=1, \quad b a_{2}^{2}(\zeta \alpha)^{-2 n}+c a_{1} \alpha^{q}=0 .
$$

Putting $t=-q-2 n$, we compute that $b=2 c^{2} \zeta^{n-q}, a_{2}^{3}=a_{1}^{3} \zeta^{-t} \alpha^{-2 t}$, $a_{1}^{3}=-\alpha^{t} /\left(8 c^{3} \zeta^{n-q}\right)$. Hence we write $\eta=\zeta^{n-q}$.

A (hopefully) more transparent description of the infinite families of solutions of (1.2) will be given in the next section.

8. The equation $2 \eta c^{2} u_{n}^{2}+c u_{q}=1$. As stated after Theorem $2, \eta$ is a root of unity (and of course $c \neq 0$ ). For arbitrary $\alpha \in \mathbb{C}$ which is non-zero and not a root of unity, and an integer root $\eta_{1}$ of $\eta$ put $w_{n}=-\frac{1}{2 \eta c}\left(\alpha^{n}+\left(\eta_{1} \alpha\right)^{-n}\right)$. Then $r=2$ and $(n, q)$ gives a solution of the equation (with $u_{n}$ replaced by $w_{n}$ ) for $q=2 n, \eta_{1}^{n}=1 / \eta$ (it is subordinate to the partition $\mathcal{P}_{1}$ defined in the preceding section).

Similarly, for $\widetilde{\alpha} \in \mathbb{C}$ as above, $\eta_{2}, \eta_{3}$ such that $\eta_{2}^{3}=\eta, \eta_{3}^{3}=1$ and an integer root $\eta_{4}$ of $\eta_{2} \eta_{3}$ put $\widetilde{w}_{n}=-\frac{1}{2 \eta_{2} c}\left(\widetilde{\alpha}^{n}+\eta_{3}\left(\eta_{4} \widetilde{\alpha}\right)^{-n}\right)$. This gives solutions $(n, q)$ for $q=-2 n, \eta_{4}^{n}=\eta_{2} \eta_{3}$ (they are subordinate to $\mathcal{P}_{2}$ ).

It follows from the formulas in Section 7 that for $u_{n}=w_{n}$ resp. $\widetilde{w}_{n}$ these pairs $(n, q)$ give all solutions subordinate to $\mathcal{P}_{1}$ resp. $\mathcal{P}_{2}$. Moreover, $\left(w_{n}\right),\left(\widetilde{w}_{n}\right)$ are the recurrence sequences leading to $t=0$. In the general case considered in Section 7, the results obtained for $\mathcal{P}_{1}$ can be formulated as $u_{n}=w_{n+t}$ (with $\eta_{1}=\zeta, t$ is determined by $\alpha^{t}=-2 a_{1} c \eta$ ), and for $\mathcal{P}_{2}: u_{n}=$ $\widetilde{w}_{3 n+t}\left(\right.$ with $\left.\widetilde{\alpha}^{3}=\alpha, \eta_{4}^{3}=\zeta, \alpha^{t}=-8 a_{1}^{3} c^{3} \eta, \eta_{2}=-\frac{\widetilde{\alpha}^{t}}{2 c a_{1}}, \eta_{3}=\widetilde{\alpha}^{2 t} \eta_{4}^{t} \frac{a_{2}}{a_{1}}\right)$. 
If $\eta \neq \pm 1$, it follows from [SS1, Prop. 1] that the intersection of the sequences $\left(w_{n}\right)$ and $\left(\widetilde{w}_{n}\right)$ contains at most finitely many solutions for $\mathcal{P}_{1}$, hence in this case the set of all solutions $(n, q)$ for $\left(w_{n}\right)$ or $\left(\widetilde{w}_{n}\right)$ consists of finitely many pairs and one infinite arithmetic progression. The same for $\left(\widetilde{w}_{n}\right)$ with $\eta \neq \eta_{2}$ or $\eta_{3} \neq 1$. However, for $\eta=\eta_{2}= \pm 1, \eta_{3}=1$, $\eta_{1}=\eta_{4}, \alpha=\widetilde{\alpha}$, we have $w_{n}=\widetilde{w}_{n}$ and we get two infinite arithmetic progressions in the set of solutions. For a general sequence $\left(u_{n}\right)$, we can summarize our computations by saying that apart from finitely many terms the set of solutions for $\left(u_{n}\right)$ always belongs to the intersection of $\left(u_{n}\right)$ with one of the "basic" sequences $\left(w_{n}\right)$ or $\left(\widetilde{w}_{n}\right)$ (for appropriate $\alpha$ or $\widetilde{\alpha}$ and $\eta_{i}$ ).

Put

$$
w_{n}^{\prime}=-\frac{1}{2 \eta c}\left(\alpha^{n}+\eta \alpha^{-n}\right), \quad w_{n}^{\prime \prime}=-\frac{1}{2 \eta c}\left(\alpha^{n}+\eta^{2} \alpha^{-n}\right) .
$$

If $(n, 2 n)$ belongs to the set of solutions for $\left(w_{n}\right)$ (subordinate to $\left.\mathcal{P}_{1}\right)$, it follows (from $\eta_{1}^{n}=1 / \eta$ ) that $w_{n}=w_{n}^{\prime}, w_{2 n}=w_{2 n}^{\prime \prime}$. This means that the corresponding terms of the sequence $\left(w_{n}\right)$ do not depend on the choice of the root $\eta_{1}$ of $\eta$. The rôle of $\eta_{1}$ is to filter out an arithmetic progression of integers by the condition $\eta_{1}^{n}=1 / \eta$. Choosing $\eta_{1}$ so that $\operatorname{ord}(\eta) \mid \operatorname{ord}\left(\eta_{1}\right)$ (order in the group of roots of unity) maximizes the set of solutions for $\mathcal{P}_{1}$. Similarly for $\left(\widetilde{w}_{n}\right)$ with

$$
\widetilde{w}_{n}^{\prime}=-\frac{1}{2 \eta_{2} c}\left(\widetilde{\alpha}^{n}+\frac{1}{\eta_{2}} \widetilde{\alpha}^{-n}\right), \quad \widetilde{w}_{n}^{\prime \prime}=-\frac{1}{2 \eta_{2} c}\left(\widetilde{\alpha}^{n}+\eta_{2}^{2} \widetilde{\alpha}^{-n}\right) .
$$

For solutions $(n,-2 n)$ subordinate to $\mathcal{P}_{2}$, one has $\widetilde{w}_{n}=\widetilde{w}_{n}^{\prime}, \widetilde{w}_{-2 n}=\widetilde{w}_{-2 n}^{\prime \prime}$. As above, $\eta_{4}$ serves to filter out an arithmetic progression of integers (by $\left.\eta_{4}^{n}=\eta_{2} \eta_{3}\right)$, the maximal case is obtained for $\operatorname{ord}\left(\eta_{2} \eta_{3}\right) \mid \operatorname{ord}\left(\eta_{4}\right)$.

If $\operatorname{ord}(\eta)$ is a multiple of 3 , then it is enough to consider $\left(\widetilde{w}_{n}\right)$ just for one choice of $\eta_{2}, \eta_{3}$ (i.e., for any other choice of $\eta_{2}, \eta_{3}$, the solutions for $\mathcal{P}_{2}$ will belong to the intersection of the two sequences if $\widetilde{\alpha}$ is modified by multiplication with an appropriate root of unity and $\eta_{4}$ is also chosen properly). But if 3 does not divide ord $\left(\eta_{2} \eta_{3}\right)$ (hence $3 \nmid \operatorname{ord}(\eta)$ ), the solutions for $\mathcal{P}_{2}$ will be only partially in common when changing the parameters (except for the trivial case when taking roots of $\widetilde{\alpha}$ and $\eta_{4}$ ). Along this line, take for example $\eta= \pm 1, \eta_{2} \neq \eta, \eta_{3}=\eta / \eta_{2}, \eta_{4}=\eta$. Then the corresponding sequence $\left(\widetilde{w}_{n}\right)$ (and its set of solutions for $\mathcal{P}_{2}$ ) has infinite intersection with that for $\eta_{2}=\eta, \eta_{3}=1$ when $\widetilde{\alpha}$ is replaced by $\alpha=\widetilde{\alpha} \eta_{3}$. As mentioned above, this second sequence coincides with a sequence $\left(w_{n}\right)\left(\alpha\right.$ as above, $\left.\eta_{1}=\eta\right)$. But the first sequence contains no solutions for $\mathcal{P}_{1}$.

9. The equation $a u_{h}^{k}+b u_{n}^{2}+c u_{q}=0$. According to the general assumptions of (1.1), we assume that $k>2, a, b, c \neq 0$. 
(a) Let $\eta$ be any root of unity, and put $2 \eta c^{\prime}=b / c$. Then this can be reduced to the equation of Section 8 for $(n, q)$ (with $c^{\prime}$ instead of $c$ ) as soon as $u_{h}^{k}=-2 \eta c^{2} /(a b)$. Hence if there exists such an integer $h$ and $\left(u_{n}\right)$ is of the type described in Section 8, we will get infinitely many solutions. For example, if $u_{n}=w_{n}$ (or $u_{n}=\widetilde{w}_{n}$ ), then given $a, b, c, k, \eta$ there are countably infinitely many $\alpha$ (or $\widetilde{\alpha}$ ) for which there exists an $h$ such that $u_{h}^{k}$ has the desired value.

Under the transformation used above, the partition $\mathcal{P}_{1}$ of Section 7 corresponds to a partition $\mathcal{P}_{1}^{\prime}$. It is obtained by replacing $\{(1,2), *\}$ by $\{(1,2)\} \cup A_{k}$. It arises in Case $\mathrm{A}$ for $K=0$ (see Property 10.1). In the same way, $\mathcal{P}_{2}$ corresponds to a partition $\mathcal{P}_{2}^{\prime}$. It arises from Case B with $M=0, K=-2 L$ (see Property 10.8), when interchanging the rôles of $k, m$ (to fit the equality of the title).

(b) We now assume that $k=3$, i.e., we study the equation $a u_{h}^{3}+b u_{n}^{2}+$ $c u_{q}=0$. Then there are further possibilities to get infinitely many solutions. For $r=2$, we consider the partition $\mathcal{P}_{3}=\{\{(1,1,1),(1)\},\{(1,1,2),(1,1)\}$, $\{(1,2,2),(1,2)\},\{(2,2,2),(2,2),(2)\}\}$ of $A$. This is a partition without singletons arising in Case A for $v_{2}=0,(K, L, M)=(1,1,3)$ (see Property 10.2; the rôles of $k, l$ have been interchanged). We write $u_{n}=a_{1} \alpha^{n}+a_{2} \zeta^{n}$ (as before, $\alpha \in \mathbb{C} \backslash\{0\}$ not a root of unity, $\zeta$ a root of unity). Searching for solutions subordinate to $\mathcal{P}_{3}$, by translating the sequence we can always assume that $3 h=q$ (the first of the equations below implies that if this holds for one solution, then it holds for all solutions for $\left(u_{n}\right)$ subordinate to $\left.\mathcal{P}_{3}\right)$. Then, putting $t=n-h$, one gets the equations: $a a_{1}^{2}+c=0,3 a a_{2} \zeta^{h}+b \alpha^{2 t}=0$, $3 a a_{2} \zeta^{h}+2 b \zeta^{t} \alpha^{t}=0, a a_{2}^{2}+b a_{2} \zeta^{2 t-h}+c=0$. By elementary computations, this is equivalent to: $\alpha^{t}=2 \zeta^{t}, a_{1}^{2}=a_{2}^{2} / 4=-c / a, a_{2}=-\frac{4 b}{3 a} \zeta^{2 t-h}$. This shows that equation (1.1) with $k=3$ has infinitely many solutions $(h, n, q)$ subordinate to $\mathcal{P}_{3}$ (for certain $\alpha, \zeta$ ) if and only if $\eta=\frac{4 b^{2}}{9 a c}$ is a root of unity. Then $\alpha, \zeta$ are determined as follows: take a root $\alpha_{0}$ of 2 , i.e., $\alpha_{0}^{t}=2$ for some $t \in \mathbb{Z} \backslash\{0\}$ and choose $\zeta$ so that $\zeta^{2 h_{0}-4 t}=-\eta$ for some $h_{0} \in \mathbb{Z}$ (of course, $h_{0} \neq 2 t$ for $\left.\eta \neq-1\right)$. Then put $\alpha=\alpha_{0} \zeta, \eta_{1}=\zeta^{2 t-h_{0}}, a_{2}=-\frac{4 b}{3 a} \eta_{1}$, $a_{1}= \pm a_{2} / 2$ (this implies $\left.\eta_{1}^{2}=-1 / \eta\right)$. For such a sequence $\left(u_{n}\right)$ all solutions subordinate to $\mathcal{P}_{3}$ are given by $\zeta^{h}=\zeta^{h_{0}}, n=h+t, q=3 h$. Up to translating the sequence, this gives all possibilities to have infinitely many solutions subordinate to $\mathcal{P}_{3}$.

(c) Again for $k=3$, we consider the partition $\mathcal{P}_{4}=\{\{(1,1,1),(2,2),(1)\}$, $\{(1,1,2),(1,2)\},\{(1,2,2),(1,1)\},\{(2,2,2),(2)\}\}$ of $A$. This arises in Case Bbb for $2 v_{2}=-3 v_{1},(K, L, M)=(1,-1,3)$ (see Property 10.11, again $k, l$ are interchanged). We write $u_{n}=a_{1} \alpha^{-2 n}+a_{2} \zeta^{n} \alpha^{3 n}$. This is somehow related to (b). After a translation, we can assume $3 h=q$ and then, putting $t=n+h$, some computations show that the conditions imposed by $\mathcal{P}_{4}$ are equivalent to: $\zeta^{t} \alpha^{5 t}=2, a_{1}^{2}=4 a_{2}^{2}=-\frac{4 c}{a}, a_{1}=-\frac{2 b}{3 a} \zeta^{t-2 h} \alpha^{t}$. The 
result is that there are infinitely many solutions subordinate to $\mathcal{P}_{4}$ (for some non-degenerate sequence $\left(u_{n}\right)$ with $r=2$ ) if and only if $\eta=4\left(\frac{b^{2}}{9 a c}\right)^{5}$ is a root of unity. Then $\alpha, \zeta$ are determined as follows: take a root $\alpha_{0}$ of 2 , i.e., $\alpha_{0}^{t}=2$ for some $t \in \mathbb{Z} \backslash\{0\}$ and choose $\zeta$ so that $\zeta^{20 h_{0}-8 t}=-\eta$ for some $h_{0} \in \mathbb{Z}$. Then choose $\alpha$ with $\alpha^{5} \zeta=\alpha_{0}$ and put $\eta_{1}=\zeta^{t-2 h_{0}}, a_{1}=-\frac{2 b}{3 a} \eta_{1} \alpha^{t}$, $a_{2}= \pm a_{1} / 2$. If $t$ is not a multiple of 5 , the fifth root $\alpha$ of $\alpha_{0} / \zeta$ has to be chosen so that $\alpha^{-2 t}=-\eta_{1}^{2} \frac{b^{2}}{9 a c}$ (it results from the expression for $\eta$ that the two quantities differ only by a fifth root of unity). If $t=5 t_{0}$, with $t_{0} \in \mathbb{Z}$, put $\eta_{0}=-\frac{b^{2}}{9 a c} \alpha_{0}^{2 t_{0}}$. Then $\eta_{0}^{5}=-\eta$ and we have to choose $\zeta$ so that $\zeta^{4 h_{0}-8 t_{0}}=\eta_{0}$ for some $h_{0} \in \mathbb{Z}$ (of course, $h_{0} \neq 2 t_{0}$ for $\eta_{0} \neq 1$ ). Then we can take any fifth root $\alpha$ of $\alpha_{0} / \zeta$ (and we always get $\alpha^{-2 t}=-\eta_{1}^{2} \frac{b^{2}}{9 a c}$ ). For such sequences $\left(u_{n}\right)$, all solutions subordinate to $\mathcal{P}_{4}$ are given by $\zeta^{2 h}=\zeta^{2 h_{0}}, n=t-h$, $q=3 h$.

Anticipating a little bit, let us note that in the terminology of Section 13, the solutions described in (b) and (c) come from the polynomial identity $3 x(1+x / 2)^{2}-(1+x)^{3}+\left(1+x^{3} / 4\right)=0$, while those found in (a) result from $2 x-(1+x)^{2}+\left(1+x^{2}\right)=0$. One could also use the formulas for $p_{i}, \widetilde{p}_{i}$ in Section 13(a) to derive the explicit form of the solutions as presented above.

(d) We claim that (given the results of the remaining sections), (a), (b), (c) describe all the exceptional cases for Theorem 1, i.e., all the cases of non-degenerate sequences $\left(u_{n}\right)$ with $r \geq 2$ for which equation (1.1) has infinitely many solutions $(h, n, q)$.

By Section 3, equation (1.1) has at most finitely many solutions that are subordinate to a partition $\mathcal{P}$ with singletons (i.e., they belong to the set $\bar{S}(\mathcal{P})$ as defined at the beginning of Section 11). For partitions $\mathcal{P}$ without singletons, it results from the Proposition (Section 6) that for equation (1.1), $\bar{S}(\mathcal{P})$ can be infinite only for $g=1$, and then Section 13 will give as the only possibilities Case A with $K=0$ (this is described in Property 10.1), Case B with $M=0$ (Property 10.8), and furthermore, for $l=3, m=1$, there is Case A with $v_{r}=0$ (Property 10.2) and Case Bbb (Property 10.11). The descriptions given in Properties 10.1, 10.2, 10.8, 10.11 show that in all these cases we have $r=2$ and by Section 11 (see in particular the Remark after Consequence 11.3) $f_{1}, f_{2}$ have to be constant. This leads to (a), (b), (c), finishing the proof of our claim.

10. Further properties of $\bar{\sigma}$. We now start with preparations for the proof of Theorem 1 in the case $g=1$, which will be finished in Section 13 . The basis is again Laurent's theorem (see Section 2), but as mentioned in the Introduction, for $g=1$ partitions $\mathcal{P}$ for which the groups $\mathcal{H}(\mathcal{P})$ are nontrivial exist in abundance (making it impossible to use an argument as in Section 6). The aim of the present section will be to collect further properties 
of these partitions. To make orientation easier, I start with a summary, indicating the direction of the argument. As explained at the beginning of the next section, it is enough to investigate the partitions $\mathcal{P}_{\bar{\sigma}}$, defined in Section 4 by an integer triple $(K, L, M)$ and numbers $v_{1}, \ldots, v_{r}$. Properties 10.1-10.7 will be dealing with Case A. First, we describe two degenerate cases $(K=0$ in 10.1, giving "essentially two-dimensional" partitions, and $v_{r}=0$ in 10.2, giving "asymmetric" partitions). This can happen only for $r=2$, it will need separate treatment in Section 13; the exceptions (for Case A) are among these. Among the non-degenerate partitions, there is another side-branch furnished by those partitions with $L=\operatorname{gcd}(K, L, M)$ (in most of the proof, we will assume that $\operatorname{gcd}(K, L, M)=1$ and write simply $L=1$ ). This is limited to $r=2,3$ (Property 10.4). It will lead to some nasty computations in Section 13(f),(g) when $m=1$ or $k=1$, containing some cases that come very close to generate further exceptions. For the "generic" case $L>\operatorname{gcd}(K, L, M)$ there is the important Property 10.6:M=K+L (which implies $2 \leq m<k$; see the Remark to 10.6). Then Property 10.7 gives a final distinction. The second option of 10.7 provides a further equation which has to be satisfied by the solutions subordinate to such a partition (if there are infinitely many; see 11.4) and this contradicts equations for other elements of the partition (see Section 13(b)). The first option of 10.7 implies that the numbers $v_{1}, \ldots, v_{r}$ lie sufficiently close together to make Mason's method applicable (at least for $m>2$ ); see Section 13(c). The reader who wants to get a picture of the proof of Theorem 1 in the "generic" case of type A can pass from 10.7 to the next section.

In 10.8-10.11 corresponding statements for the cases of type B are shown. There is no complete correspondence between the basic types as introduced in Section 5 (i.e., we do not have a bijective transformation associating to a partition of type A a partition of type Baa etc.), but it turns out that the properties that are relevant to the proof of Theorem 1 have natural counterparts in the other cases. This is formulated as a "transference scheme" in the final Remark of the section; it will make it possible to do most of the proof of Theorem 1 in Section 13 parallel for the various cases. 10.12, 10.13 are devoted to the cases $r=3$ and $r=2$ where a rather complete picture can be given.

We keep the assumptions of Section 5, i.e., $r \geq 2, v_{1}<\cdots<v_{r}$, and we will always assume that $K, L, M$ are given so that $\mathcal{P}_{\bar{\sigma}}$ has no singletons. In 10.1-10.2 we describe some limiting cases for the parameters. Then (10.3, 10.4) we start with a more detailed investigation of Case A.

10.1. In Case A (Property 5.2 and (5.1)), we have $K>0$ with the following exception: $r=2, l=2, m=1, M=2 L, K=0, v_{2}=-v_{1}$. 
Proof. If $K=0$, then by (5.2), $v_{r} \geq v_{2}>0$ and we have $K k v_{r}=$ $L(l-1) v_{r}+L v_{r-1}$ (see also 10.2 below). (5.1) implies $l>m \geq 1$ and it follows from the preceding equation and (5.2) that $v_{r-1}-v_{2}=(l-1)\left(v_{1}-v_{r}\right)<0$. This implies $r=2$ and then $l=2, m=1, v_{2}=-v_{1}$. Finally, by (5.1), $M=2 L$.

10.2. In Case $\mathrm{A}$, we have $v_{r}>0$ with the following exception: $r=2$, $k=l-1, m \mid l, K=L, M=\frac{l}{m} L, v_{2}=0, l \geq 3$. For $v_{r}>0$, we have

$$
K k v_{r}=L(l-1) v_{r}+L v_{r-1} .
$$

Proof. If $v_{r}>0$, then (10.1) follows from (5.2) by considering the reflected sequence $v_{i}^{*}=-v_{r+1-i}$. Now assume that $v_{r} \leq 0 . K=0$ has already been excluded in 10.1. Thus $K>0$. Considering the maximal values (using Property 5.1 and $(5.1)),(r, \ldots, r)_{k}$ gives a singleton unless $v_{r}=0$. For $v_{r}=0$ and $K<L(\operatorname{resp} . K>L),(r-1, r, \ldots)_{k}\left(\operatorname{resp} .(r-1, r, \ldots)_{l}\right)$ gives a singleton. Thus $K=L$. Then (5.1) implies $k<l$ and (5.2) gives $L\left(v_{2}-v_{1}\right)=$ $L(k-l) v_{1}$. Since $0<v_{2}-v_{1} \leq-v_{1}$, it follows that $v_{2}=0, k-l=-1$ and in particular $r=2$. Then it is easily seen that $\bar{\sigma}\left(A_{l}\right)=\left\{-L l v_{1}, L l v_{1}\right\} \cup \bar{\sigma}\left(A_{k}\right)$. Hence, if $m>1$, we must have $\bar{\sigma}(\ldots, 1,2)_{m} \in \bar{\sigma}\left(A_{l}\right)$. This gives $M v_{1}=L t v_{1}$ for some $t \in \mathbb{N}$, thus $L l=M m$ implies $l=m t$, hence $m \mid l$. In particular, $l \geq 3$, since $m \neq k, l$.

REMARK. (5.2) can be rewritten as $(L l-K k)\left(-v_{1}\right)=L\left(v_{2}-v_{1}\right)$, similarly for (10.1). This gives for Case A with $v_{r}>0$ :

$$
(L l-K k)\left(v_{r}-v_{1}\right)=L\left(v_{2}-v_{1}\right)+L\left(v_{r}-v_{r-1}\right) .
$$

10.3. In Case $\mathrm{A}$, we have for $r=3: K k=L(l-1)$ and $v_{2}=0$. For $r=2, v_{2}>0$ implies $K k=L(l-2)$ and $v_{2}=-v_{1}$.

Proof. The first equality for the case $r=3$ follows immediately from (10.2) (and Property 10.2). Inserting this to (5.2) gives $v_{2}=0$. The case $r=2$ is treated in the same way.

10.4. In Case A with $r>3$, we have $0<L l-K k<L$.

Proof. By (10.2).

The exceptional cases of 10.1, 10.2 above (and also those of 10.8, 10.11) yield examples of partitions $\mathcal{P}$ of $A$ without singletons and non-trivial groups $\mathcal{H}(\mathcal{P})$ (with $g=1, r=2$ ). 10.12 will give such a (somewhat exceptional) example for $r=3$.

In the remaining properties, we will always assume that $K, L, M \in$ $\mathbb{Z}, v_{1}, \ldots, v_{r} \in \mathbb{Q}$, and it is no restriction to assume (by scaling) that $\operatorname{gcd}(K, L, M)=1$. Then Property 10.4 can be reformulated as $1 \leq L l-K k$ $<L$. 
To get further information about the partitions $\mathcal{P}_{\bar{\sigma}}$ without singletons (for the "generic case", in particular for $r>3$ ), we will investigate the second smallest value of $\sigma$ on $A_{k}$ and $A_{m}$. This will lead to a further relation between $K, L, M$. In 10.6, 10.7 this will be done for Case A.

In place of the two-dimensional arguments in the proof of the Proposition, we will now exploit divisibility properties (see also the proofs in [SS1, Sec. 6-9]). For a prime $p,||_{p}$ denotes the usual $p$-adic absolute value. We put $v_{i}^{\prime}=v_{i}-v_{1}$ and we will assume from now on that $v_{2}^{\prime}, \ldots, v_{r}^{\prime} \in \mathbb{Z}$ (which is always achievable by scaling). Property 10.5 exhibits further points of $A_{k}$ whose values under $\sigma$ are not attained at another point of $A_{k}$ ("isolated values"). The minimality statement for $\sigma\left(\ldots, 1, j_{0}\right)_{k}$ generalizes Property 5.1 and holds in fact for any $j_{0} \in\{1, \ldots, r\}$.

10.5. For a prime $p$ assume that $j_{0}$ satisfies $\left|v_{j}^{\prime}\right|_{p}<\left|v_{j_{0}}^{\prime}\right|_{p}$ for all $j<j_{0}$. Then $\left|\sigma(\mathbf{i})-k v_{1}\right|_{p} \leq\left|v_{j_{0}}^{\prime}\right|_{p}$ for all $\mathbf{i}=\left(i_{1}, \ldots, i_{k}\right) \in A_{k}$ with $i_{k} \leq j_{0}$, the inequality being strict for $i_{k}<j_{0}$.

$\sigma\left(\ldots, 1, j_{0}\right)_{k}$ is the smallest value of $\sigma$ among those $\mathbf{i}=\left(i_{1}, \ldots, i_{k}\right) \in A_{k}$ for which $i_{k} \geq j_{0}$. This value is not attained at another point of $A_{k}$.

Proof. This follows immediately from the definition of $\sigma((4.1))$.

10.6. In Case A with $L>1$, we have $M=K+L$. In particular, $\bar{\sigma}(\ldots, 1,2)_{m}=\bar{\sigma}(\ldots, 1,2)_{k}$.

Note that by 10.4 this applies always for $r>3$.

Proof. First, we want to show that $\operatorname{gcd}(K, L)=1$. Assume $p$ is a prime such that $|K|_{p},|L|_{p}<1$. Then our general assumption $\operatorname{gcd}(K, L, M)=1$ implies $|M|_{p}=1$. Now, let $s_{0}$ be the smallest index such that $\left|v_{s}^{\prime}\right|_{p} \leq\left|v_{s_{0}}^{\prime}\right|_{p}$ for all $s$. Then, since by $(5.2),(K k-L l) v_{1}=L\left(v_{2}-v_{1}\right)$ and $\left|L\left(v_{2}-v_{1}\right)\right|_{p}$ $<\left|v_{s_{0}}^{\prime}\right|_{p}$, by 10.5 we get $\left|\bar{\sigma}(\mathbf{i})-L l v_{1}\right|_{p}<\left|v_{s_{0}}^{\prime}\right|_{p}$ for all $\mathbf{i} \in A_{k} \cup A_{l}$. On the other hand, $L l=M m$ (by (5.1)), hence $\left|\bar{\sigma}\left(\ldots, 1, s_{0}\right)_{m}-L l v_{1}\right|_{p}=\left|v_{s_{0}}^{\prime}\right|_{p}$. Consequently, by $10.5,\left(\ldots, 1, s_{0}\right)_{m}$ would be a singleton. This proves that $\operatorname{gcd}(K, L)=1$.

Since $L>1$, there is a prime $p$ such that $p \mid L$. Then $|K|_{p}=1$. Define $s_{0}$ as above and consider $\left(\ldots, 1, s_{0}\right)_{k}$. As above, we get $\bar{\sigma}\left(\ldots, 1, s_{0}\right)_{k} \notin$ $\bar{\sigma}\left(A_{l}\right)$, hence to avoid singletons and using $10.5, \bar{\sigma}\left(\ldots, 1, s_{0}\right)_{k}$ must belong to $\bar{\sigma}\left(A_{m}\right)$. This entails $|M|_{p}=1$ and $\bar{\sigma}\left(\ldots, 1, s_{0}\right)_{k} \geq \bar{\sigma}\left(\ldots, 1, s_{0}\right)_{m}$. Then the same argument for $\left(\ldots, 1, s_{0}\right)_{m}$ gives $\bar{\sigma}\left(\ldots, 1, s_{0}\right)_{k}=\bar{\sigma}\left(\ldots, 1, s_{0}\right)_{m}$. This means that $L v_{2}^{\prime}+K v_{s_{0}}^{\prime}=M v_{s_{0}}^{\prime}$. If $s_{0}=2$, the result follows. For later use, note also that since we have shown that $|M|_{p}=1$ for all primes dividing $L$, it follows that $\operatorname{gcd}(L, M)=1$.

Now assume that $s_{0}>2$. Since $(M-K) v_{s_{0}}^{\prime}=L v_{2}^{\prime}$ and $0<v_{2}^{\prime}<$ $\cdots<v_{r}^{\prime}$, we conclude that $K<M$ and that $(M-K) v_{s}^{\prime}<L v_{2}^{\prime}$ for $s<s_{0}$. This means that $\bar{\sigma}(\ldots, 1, s)_{m}<\bar{\sigma}(\ldots, 1, s)_{k}$ for $1 \leq s<s_{0}$. 
For $s=2$, this implies $\bar{\sigma}(\ldots, 1,2)_{m} \in \bar{\sigma}\left(A_{l}\right)$. Then, as in the preceding paragraph, we get $\bar{\sigma}(\ldots, 1,2)_{m}=\bar{\sigma}\left(\ldots, 1, s_{1}\right)_{l}$, where $s_{1}$ is the smallest index such that $\left|v_{s_{1}}^{\prime}\right|_{p}>\left|v_{2}^{\prime}\right|_{p}$. If $s_{1}<s_{0}$, we can repeat this argument and get $\bar{\sigma}\left(\ldots, 1, s_{1}\right)_{m}=\bar{\sigma}\left(\ldots, 1, s_{2}\right)_{l}$, where $s_{2}$ is the smallest index such that $\left|v_{s_{2}}^{\prime}\right|_{p}>\left|v_{s_{1}}^{\prime}\right|_{p}$ and so on. Finally, $s_{j}=s_{0}$ (where $j \geq 1$ ). We have $M v_{2}^{\prime}=$ $L v_{s_{1}}^{\prime}, M v_{s_{i}}^{\prime}=L v_{s_{i+1}}^{\prime}(1 \leq i<j)$ and this gives $v_{s_{0}}^{\prime}=v_{s_{j}}^{\prime}=(M / L)^{j} v_{2}^{\prime}$. Combined with the earlier equation, we get $(M-K)(M / L)^{j}=L$. It would follow that $M^{j} \mid L^{j+1}$, contradicting $\operatorname{gcd}(L, M)=1, L<M$.

REMARK. The proof shows that for any prime $p$ with $|L|_{p}<1$ we have $\left|v_{i}^{\prime}\right|_{p} \leq\left|v_{2}^{\prime}\right|_{p}$ for all $i$. Properties 5.2 and 10.6 show for Case A with $L>1$ that $\mathcal{H}\left(\mathcal{P}_{\bar{\sigma}}\right)$ is cyclic and that $(K, L, M)$ has to be a (rational) multiple of $(l-m, m, l)$ (in particular, $m \geq 2$ ). Furthermore, $m<k$ (indeed, we have $v_{r}>0$ by 10.2 , and $(10.2)$ gives $L(l-2) \leq K k$, which entails $m(l-2) \leq$ $(l-m) k)$.

10.7. In Case $\mathrm{A}$ with $L>1$ there are the following two alternatives: either $v_{2}^{\prime} \mid v_{i}^{\prime}$ for all $i$ or there exists an $i$ such that $\left\{(\ldots, 1, i)_{k},(\ldots, 1, i)_{l}\right\}$ belongs to $\mathcal{P}_{\bar{\sigma}}$. In the second case, we have $K<L, L v_{2}^{\prime}=(L-K) v_{i}^{\prime}$ and $\operatorname{gcd}\left(v_{2}^{\prime}, v_{i}^{\prime}\right) \mid v_{j}^{\prime}$ for all $j$.

The two alternatives are easily seen to be exclusive, but the final properties are also compatible with the first alternative $v_{2}^{\prime} \mid v_{i}^{\prime}$ for $K=L-1$.

Proof. If the first alternative does not hold, then by the preceding Remark, there is a prime $p$ such that $p \nmid L$ and $\left|v_{i}^{\prime}\right|_{p}>\left|v_{2}^{\prime}\right|_{p}$ for some $i$. Let $s_{0}$ be the smallest index with $\left|v_{s_{0}}^{\prime}\right|_{p}=\max _{i}\left|v_{i}^{\prime}\right|_{p}$. Then by 10.5 , the value $\bar{\sigma}\left(\ldots, 1, s_{0}\right)_{l}$ is attained nowhere else on $A_{l}$. Since $L<M$, it is not attained on $A_{m}$. Hence, $\bar{\sigma}\left(\ldots, 1, s_{0}\right)_{l} \in \bar{\sigma}\left(A_{k}\right)$. This implies $p \nmid K$ and then, as in the first part of the proof of 10.6, it follows that $\bar{\sigma}\left(\ldots, 1, s_{0}\right)_{l}=\bar{\sigma}\left(\ldots, 1, s_{0}\right)_{k}$. This means that $L v_{s_{0}}^{\prime}=L v_{2}^{\prime}+K v_{s_{0}}^{\prime}$. In particular, $s_{0}$ does not depend on $p$ and this implies that $\left|v_{j}^{\prime}\right|_{p} \leq \max \left(\left|v_{2}^{\prime}\right|_{p},\left|v_{s_{0}}^{\prime}\right|_{p}\right)$ for all $j$ and all primes $p$. It also follows from the construction of $s_{0}$ that the value $\bar{\sigma}\left(\ldots, 1, s_{0}\right)_{k}$ is not attained at a third point of $A$, i.e., $\left\{\left(\ldots, 1, s_{0}\right)_{k},\left(\ldots, 1, s_{0}\right)_{l}\right\} \in \mathcal{P}_{\bar{\sigma}}$.

Next (10.8-10.11) we prove corresponding statements for the cases of type B.

10.8. In Case B (Properties 5.3, 5.5), we have $K, L, M \neq 0$ with the following exceptions: $r=2, M=0, K=-\frac{l}{k} L, v_{2}=-v_{1}$ and either (a) $l=2, k=1$ (Case Baa) or (b) $k=2, l=1$ (Case Bab).

As already remarked at the end of Section 5, the two options (a), (b) are related by interchanging $k, l$ and multiplying $K, L$ by -1 .

Proof. This is obvious in Case $\mathrm{Bb}$ (Property 5.5), since $\mathrm{Ll}=\mathrm{Mm}>$ $-K k, K<0$. In Case Baa (Property 5.4), $M=0$ leads to (a) as in the 
proof of Property 10.1, using $v_{r}=-v_{1}, v_{r-1}=-v_{2}$. As remarked above, Case Bab with $M=0$ can be reduced to Baa, giving (b).

Remark. Equality (10.2) has obvious analogues, e.g., in Case Bba we have by Property 5.6,

$$
(L l+K k)\left(v_{r}-v_{1}\right)=L\left(v_{2}-v_{1}\right)+L\left(v_{r}-v_{r-1}\right) .
$$

The counterparts of Properties 10.3, 10.4 in Case Bba are as follows: $1 \leq L l+K k<L$ for $r>3, K k=-L(l-1)$ for $r=3$ and $K k=-L(l-2)$ for $r=2$. Similarly for Cases Baa and Bab. This is part of the transference scheme among the cases that is described in the Remark after 10.13.

10.9. In Case Bba with $L>1$, we have $v_{1}=-v_{r}, v_{2}=-v_{r-1}$ and $M=L-K$. In particular, $\bar{\sigma}(\ldots, 1,2)_{m}=\bar{\sigma}(r-1, r, \ldots)_{k}$.

Note that by the statement after $\left(10.2^{\prime}\right)$ this applies always for $r>3$.

Proof. The basic strategy is similar to that of 10.6. But since in this case $K<0$, the maxima and minima of $\sigma$ on the parts of $A$ get interrelated and the details are becoming more complex. We put $v_{i}^{\prime \prime}=v_{r}-v_{i}=v_{r}^{\prime}-v_{i}^{\prime}$. Note that $v_{i}^{\prime \prime}>0$ for $i<r$ and that for any prime $p$, we have $\max _{i}\left|v_{i}^{\prime}\right|_{p}=$ $\max _{i}\left|v_{i}^{\prime \prime}\right|_{p}$.

(a) Take a prime $p$ with $|L|_{p}<1$. Let $s_{0}$ be the smallest value and $t_{0}$ be the largest value such that $\left|v_{s_{0}}^{\prime}\right|_{p}=\left|v_{t_{0}}^{\prime \prime}\right|_{p}=\max _{i}\left|v_{i}^{\prime}\right|_{p}$.

First we want to show that $\operatorname{gcd}(K, L)=1$. Assume that $|K|_{p}<1$. Then our general assumption $\operatorname{gcd}(K, L, M)=1$ implies $|M|_{p}=1$. As in 10.6, it follows that the values $\bar{\sigma}\left(\ldots, 1, s_{0}\right)_{m}, \bar{\sigma}\left(t_{0}, r, \ldots\right)_{m}$ are isolated in $\bar{\sigma}\left(A_{m}\right)$ and do not belong to $\bar{\sigma}\left(A_{k}\right) \cup \bar{\sigma}\left(A_{l}\right)$. Hence $\mathcal{P}_{\bar{\sigma}}$ would have a singleton.

(b) Fixing now any prime $p$ with $|L|_{p}<1$, we get $|K|_{p}=1$ by (a). Then it follows as in 10.6 that in order to avoid singletons, we must have $\bar{\sigma}\left(\ldots, 1, s_{0}\right)_{m}=\bar{\sigma}\left(t_{0}, r, \ldots\right)_{k}$ and $\bar{\sigma}\left(\ldots, 1, s_{0}\right)_{k}=\bar{\sigma}\left(t_{0}, r, \ldots\right)_{m}$. In particular, $|M|_{p}=1$, which entails $\operatorname{gcd}(L, M)=1$. Note that up to this point, a similar procedure can be used in Cases Baa, Bab (as long as $L>1$ resp. $K<-1$ ) and in Cases Bbb and Bbc (when $L>1$ ).

Getting back to Case Bba, the identities for $\bar{\sigma}$ result by Property 5.6 in the following equations:

$$
M v_{s_{0}}^{\prime}=L v_{2}^{\prime}-K v_{t_{0}}^{\prime \prime}, \quad M v_{t_{0}}^{\prime \prime}=L v_{r-1}^{\prime \prime}-K v_{s_{0}}^{\prime} .
$$

Addition gives $(M+K)\left(v_{s_{0}}^{\prime}+v_{t_{0}}^{\prime \prime}\right)=L v_{2}^{\prime}+L v_{r-1}^{\prime \prime}$, and since the right side of this equation is positive, it follows that $M>-K$.

(c) For $1 \leq s, t \leq r$, we get from (10.3) and Property 5.6 the following identities:

$$
\begin{array}{r}
M\left(\bar{\sigma}(\ldots, 1, s)_{m}-\bar{\sigma}(t, r, \ldots)_{k}\right)+(-K)\left(\bar{\sigma}(\ldots, 1, s)_{k}-\bar{\sigma}(t, r, \ldots)_{m}\right) \\
=\left(M^{2}-K^{2}\right)\left(v_{s}-v_{s_{0}}\right),
\end{array}
$$




$$
\begin{array}{r}
(-K)\left(\bar{\sigma}(\ldots, 1, s)_{m}-\bar{\sigma}(t, r, \ldots)_{k}\right)+M\left(\bar{\sigma}(\ldots, 1, s)_{k}-\bar{\sigma}(t, r, \ldots)_{m}\right) \\
=\left(M^{2}-K^{2}\right)\left(v_{t_{0}}-v_{t}\right) .
\end{array}
$$

This has the consequence (using $M^{2}-K^{2}>0$ ) that if $\bar{\sigma}(\ldots, 1, s)_{m} \geq$ $\bar{\sigma}(t, r, \ldots)_{k}$ and either $1 \leq s<s_{0}$ or $t_{0}<t \leq r$, then $\bar{\sigma}(\ldots, 1, s)_{k}<$ $\bar{\sigma}(t, r, \ldots)_{m}$. In particular, since $L<M$, there cannot exist a pair $s, t$ with $1 \leq s<s_{0}, \bar{\sigma}(\ldots, 1, s)_{l}=\bar{\sigma}(t, r, \ldots)_{k}$ and $\bar{\sigma}(\ldots, 1, s)_{k}=\bar{\sigma}(t, r, \ldots)_{l}$. Furthermore, $1 \leq s<s_{0}$ and $\bar{\sigma}(\ldots, 1, s)_{m}=\bar{\sigma}(t, r, \ldots)_{k}$ implies $t>t_{0}$.

(d) We will now show that $s_{0}=2, t_{0}=r-1$ leads to the desired conclusion of 10.9. This settles the case $r=2$ (where of course a more direct argument would be possible). The remaining parts (e)-(h) of the proof will be devoted to excluding the other possibilities for $s_{0}, t_{0}$.

Now, $s_{0}=2$ implies $(M-L) v_{2}^{\prime}=-K v_{r-1}^{\prime \prime}$ by (10.3), and $t_{0}=r-1$ implies $(M-L) v_{r-1}^{\prime \prime}=-K v_{2}^{\prime}$. Then it follows that $(M-L)^{2}=K^{2}$, hence (recall that $K<0<M-L$ ) we get $M-L=-K$ and $v_{2}^{\prime}=v_{r-1}^{\prime \prime}$. Addition of the identities in Property 5.6 results in $(L l-K k)\left(v_{1}+v_{r}\right)=0$. This gives $v_{1}=-v_{r}$ and (using $v_{2}^{\prime}=v_{r-1}^{\prime \prime}$ ) $v_{2}=-v_{r-1}$.

(e) Assume that $s_{0}>2, t_{0}=r-1$. Then $\bar{\sigma}(r-1, r, \ldots)_{k}=\bar{\sigma}\left(\ldots, 1, s_{0}\right)_{m}$ and it follows that the open interval $] \bar{\sigma}(\ldots, 1,2)_{l}, \bar{\sigma}\left(\ldots, 1, s_{0}\right)_{m}[$ has empty intersection with $\bar{\sigma}\left(A_{k}\right)$. Hence we can make the same procedure as in the last paragraph of the proof of Property 10.6 (alternatively, the argument in (f) could be adapted). One gets a sequence of indices $2<s_{1}<\cdots<s_{j}=s_{0}$ with $M v_{2}^{\prime}=L v_{s_{1}}^{\prime}, M v_{s_{i}}^{\prime}=L v_{s_{i+1}}^{\prime}$, resulting in $v_{s_{0}}^{\prime}=(M / L)^{j} v_{2}^{\prime}$. Then (10.3) with $t_{0}=r-1$ gives

$$
(M-L)\left(M^{j+1}-L^{j+1}\right) v_{2}^{\prime}=(M-L)(-K) L^{j} v_{r-1}^{\prime \prime}=K^{2} L^{j} v_{s_{0}}^{\prime}=K^{2} M^{j} v_{2}^{\prime}
$$

and this contradicts $\operatorname{gcd}(L, M)=1$ from (b). By symmetry, one can also exclude $s_{0}=2, t_{0}<r-1$.

(f) Assume that $s_{0}>2$ and $t_{0}<r-1$. Let $s_{1}$ be the minimal index and $t_{1}$ be the maximal index with $\left|v_{s_{1}}^{\prime}\right|_{p},\left|v_{t_{1}}^{\prime \prime}\right|_{p} \geq\left|L v_{s_{0}}^{\prime}\right|_{p}$ (recall that $\left|v_{s_{0}}^{\prime}\right|_{p}=\left|v_{t_{0}}^{\prime \prime}\right|_{p}$ by (a)). Clearly, $2 \leq s_{1} \leq s_{0}$ and $t_{0} \leq t_{1} \leq r-1$. As a first step, we claim that either $\bar{\sigma}\left(t_{0}, r, \ldots\right)_{l}=\bar{\sigma}\left(t_{1}, r, \ldots\right)_{m}$ or $\bar{\sigma}\left(\ldots, 1, s_{0}\right)_{l}=\bar{\sigma}\left(\ldots, 1, s_{1}\right)_{m}$ (in fact, we are aiming to show in (f)-(h) that $s_{1}<s_{0}, t_{1}>t_{0}$ and that both equalities hold).

By a similar reasoning as before $(10.5,10.6)$, the values $\bar{\sigma}\left(t_{1}, r, \ldots\right)_{k}$, $\bar{\sigma}\left(\ldots, 1, s_{0}\right)_{l}, \bar{\sigma}\left(\ldots, 1, s_{1}\right)_{m}$ are isolated in $\bar{\sigma}\left(A_{k}\right), \bar{\sigma}\left(A_{l}\right), \bar{\sigma}\left(A_{m}\right)$ respectively, and (using also $s_{0}>2$ ) at least two of them have to be equal and if the third value is not equal it has to be larger. Hence, if we assume $\bar{\sigma}\left(\ldots, 1, s_{0}\right)_{l} \neq$ $\bar{\sigma}\left(\ldots, 1, s_{1}\right)_{m}$, then either $\bar{\sigma}\left(t_{1}, r, \ldots\right)_{k}=\bar{\sigma}\left(\ldots, 1, s_{0}\right)_{l}<\bar{\sigma}\left(\ldots, 1, s_{1}\right)_{m}$ or $\bar{\sigma}\left(t_{1}, r, \ldots\right)_{k}=\bar{\sigma}\left(\ldots, 1, s_{1}\right)_{m}<\bar{\sigma}\left(\ldots, 1, s_{0}\right)_{l}$. We see that in both cases $\bar{\sigma}\left(t_{1}, r, \ldots\right)_{k} \leq \bar{\sigma}\left(\ldots, 1, s_{1}\right)_{m}$ and from (b) that in both cases $t_{1}>t_{0}$. By (c), we get $\bar{\sigma}\left(t_{1}, r, \ldots\right)_{m}>\bar{\sigma}\left(\ldots, 1, s_{1}\right)_{k}$. Hence, considering now the opposite 
triple $\bar{\sigma}\left(\ldots, 1, s_{1}\right)_{k}, \bar{\sigma}\left(t_{0}, r, \ldots\right)_{l}, \bar{\sigma}\left(t_{1}, r, \ldots\right)_{m}$, it follows that $\bar{\sigma}\left(t_{0}, r, \ldots\right)_{l}=$ $\bar{\sigma}\left(t_{1}, r, \ldots\right)_{m}$.

(g) We keep the notation of (f). Assuming $s_{0}>2, t_{0}<r-1$ and $\bar{\sigma}\left(t_{0}, r, \ldots\right)_{l}=\bar{\sigma}\left(t_{1}, r, \ldots\right)_{m}$, we claim that $\bar{\sigma}\left(\ldots, 1, s_{0}\right)_{l}<\bar{\sigma}\left(t_{1}, r, \ldots\right)_{k}$. The assumption gives $L v_{t_{0}}^{\prime \prime}=M v_{t_{1}}^{\prime \prime}$. Combining with (10.3) and using $L<M$, we get

$$
\begin{aligned}
\bar{\sigma}\left(\ldots, 1, s_{0}\right)_{p}-L l v_{1} & =L v_{s_{0}}^{\prime}=\frac{L^{2}}{M} v_{2}^{\prime}-\frac{L K}{M} v_{t_{0}}^{\prime \prime} \\
& <L v_{2}^{\prime}-K v_{t}^{\prime \prime}=\bar{\sigma}\left(t_{1}, r, \ldots\right)_{k}-L l v_{1} .
\end{aligned}
$$

(h) Assuming still $s_{0}>2, t_{0}<r-1$, it follows from (g) and the arguments in (f) that $\bar{\sigma}\left(t_{0}, r, \ldots\right)_{l}=\bar{\sigma}\left(t_{1}, r, \ldots\right)_{m}$ implies $\bar{\sigma}\left(\ldots, 1, s_{0}\right)_{l}=\bar{\sigma}\left(\ldots, 1, s_{1}\right)_{m}$ and by symmetry, the converse holds as well. Hence, again by (f), both equalities should be true, giving $M v_{s_{1}}^{\prime}=L v_{s_{0}}^{\prime}, M v_{t_{1}}^{\prime \prime}=L v_{t_{0}}^{\prime \prime}$, in particular $s_{1}<s_{0}, t_{1}>t_{0}$.

Let $p_{1}$ be a prime with $|M|_{p_{1}}<1$. By symmetry, we can assume that $\left|v_{s_{0}}^{\prime}\right|_{p_{1}} \geq\left|v_{t_{0}}^{\prime \prime}\right|_{p_{1}}\left(s_{0}, t_{0}\right.$ are still the old ones). Put $\delta=\left|v_{s_{0}}^{\prime}\right|_{p_{1}}$. From the relations above (and since $|L|_{p_{1}}=1$ by (b)), we get $\left|v_{s_{1}}^{\prime}\right|_{p_{1}}>\delta$, in particular $\delta<1$. The equalities (10.3) give $\left|v_{2}^{\prime}\right|_{p_{1}},\left|v_{r-1}^{\prime \prime}\right|_{p_{1}} \leq \delta$ (this implies $s_{1}>2$, hence $r>3$ ). Let $\widetilde{s}$ be the smallest index and $\widetilde{t}$ be the largest index for which $\left|v_{\tilde{s}}^{\prime}\right|_{p_{1}},\left|v_{\tilde{t}}^{\prime \prime}\right|_{p_{1}}>\delta$ (existence of $\widetilde{t}$ follows from $v_{s_{1}}^{\prime}=v_{1}^{\prime \prime}-v_{s_{1}}^{\prime \prime}$ ). Then $2<\widetilde{s} \leq s_{1}$ and $\widetilde{t}<r-1$. Again (by 10.5) we get an isolated value $\bar{\sigma}(\ldots, 1, \widetilde{s})_{l}$ in $\bar{\sigma}\left(A_{l}\right)$ and by minimality of $\widetilde{s}$ (and since $M>L$ ), it cannot belong to $\bar{\sigma}\left(A_{m}\right)$. Hence, it has to belong to $\bar{\sigma}\left(A_{k}\right)$ and, since $\widetilde{s}>2$, it follows from maximality of $\widetilde{t}$ that $\bar{\sigma}(\widetilde{t}, r, \ldots)_{k} \leq \bar{\sigma}(\ldots, 1, \widetilde{s})_{l}<\bar{\sigma}(\ldots, 1, \widetilde{s})_{m}$. Then by (c), $\bar{\sigma}(\ldots, 1, \widetilde{s})_{k}<\bar{\sigma}(\widetilde{t}, r, \ldots)_{m}<\bar{\sigma}(\widetilde{t}, r, \ldots)_{l}$. But, applying an argument as above now to $(\widetilde{t}, r, \ldots)_{l}$, it would follow that this gives a singleton for $\mathcal{P}_{\bar{\sigma}}$, providing the final contradiction.

REMARK. The proof shows that for any prime $p$ with $|L|_{p}<1$, we have $\left|v_{i}^{\prime}\right|_{p},\left|v_{i}^{\prime \prime}\right|_{p} \leq\left|v_{2}^{\prime}\right|_{p}\left(=\left|v_{r-1}^{\prime \prime}\right|_{p}\right)$ for all $i$.

10.10. In Case Baa with $L>1$, we have $M=-L-K$, in particular $\bar{\sigma}(\ldots, 1,2)_{m}=\bar{\sigma}(r-1, r, \ldots)_{k}$. In Case Bab with $K<-1$, we have $M=$ $L+K, \bar{\sigma}(\ldots, 1,2)_{l}=\bar{\sigma}(\ldots, 1,2)_{m}$.

Note that the additional conditions $(L>1$, resp. $K<-1)$ are always fulfilled for $r>3$.

Proof. This is similar to 10.6, 10.9, but easier than 10.9, since Properties 5.3 and 5.4 already contain the symmetry information $v_{2}^{\prime}=v_{r-1}^{\prime \prime}$. Starting as in (a), (b) of the proof of 10.9, it follows immediately from the equations corresponding to (10.3) that $v_{s_{0}}^{\prime}=v_{t_{0}}^{\prime \prime}$. Then one can do the construction from the last paragraph of the proof of 10.6 symmetrically on both sides. 
If $r>3$, then $M>0$ by 10.8 and $L>1$ in Case Baa (resp. $K<-1$ for Bab) follows from the analogues of (10.2) and $\left(10.2^{\prime}\right)$.

Remark. Statements similar to 10.7 (with analogous proofs) are true in the cases of type B. For Case Baa with $L>1$, the alternative to $v_{2}^{\prime} \mid v_{i}^{\prime}$ for all $i$ is the existence of an $i$ such that either $\left\{(\ldots, 1, i)_{l},(\ldots, 1, i)_{m}\right\} \in \mathcal{P}_{\bar{\sigma}}$ or $\left\{(i, r, \ldots)_{l},(i, r, \ldots)_{m}\right\} \in \mathcal{P}_{\bar{\sigma}}$. Then $M<L, L v_{2}^{\prime}=(L-M) v_{i}^{\prime}$ (resp. $L v_{2}^{\prime}=$ $\left.(L-M) v_{i}^{\prime \prime}\right)$.

In Case Bab with $K<-1$ (resp. Bba with $L>1$ ), the alternative to $v_{2}^{\prime} \mid v_{i}^{\prime}$ for all $i$ is the existence of $i, j$ such that $v_{i}^{\prime}=v_{j}^{\prime \prime},\left\{(\ldots, 1, i)_{k},(j, r, \ldots)_{m}\right\} \in \mathcal{P}_{\bar{\sigma}}$ and $\left\{(j, r, \ldots)_{k},(\ldots, 1, i)_{m}\right\} \in \mathcal{P}_{\bar{\sigma}}$. This implies $M<-K$ and $(-K-M) v_{i}^{\prime}=$ $(-K) v_{2}^{\prime}$ (resp. for Bba: $\left\{(\ldots, 1, i)_{k},(j, r, \ldots)_{l}\right\},\left\{(j, r, \ldots)_{k},(\ldots, 1, i)_{l}\right\} \in \mathcal{P}_{\bar{\sigma}}$, $L>-K$ and $\left.(K+L) v_{i}^{\prime}=L v_{2}^{\prime}\right)$.

10.11. The only instances of Case Bbb are $r=2, k=l-1, m \mid l$, $K=-L, M=\frac{l}{m} L, v_{2}=-\frac{l}{k} v_{1}, l \geq 3$.

Interchanging $v_{1}, v_{2}$ in the formulas above describes Case Bbc.

Proof (see also [G, pp. 61-66]). Again we assume that $\operatorname{gcd}(K, L, M)$ $=1$. First we claim that $K=-1$. Otherwise, let $p$ be a prime with $p \mid K$. Then, defining $s_{0}$ as in the proof of 10.6, we have now excluded $A_{k}$ from the competition and the argument from there would give $\bar{\sigma}\left(\ldots, 1, s_{0}\right)_{l}=$ $\bar{\sigma}\left(\ldots, 1, s_{0}\right)_{m}$, contradicting $L<M$.

Next we claim that $L=1$. Otherwise, taking $p$ with $p \mid L$, define $s_{0}, t_{0}$ as in part (a) of the proof of 10.9. The analogues of (10.3) in Case Bbb are $M v_{s_{0}}^{\prime}=-K v_{t_{0}}^{\prime \prime}, M v_{t_{0}}^{\prime \prime}=L v_{r-1}^{\prime \prime}-K v_{s_{0}}^{\prime}$. Since $K=-1$, this gives $(M-1)\left(v_{s_{0}}^{\prime}+v_{t_{0}}^{\prime \prime}\right)=L v_{r-1}^{\prime \prime}$. Since $v_{s_{0}}^{\prime}+v_{t_{0}}^{\prime \prime}>v_{r-1}^{\prime \prime}$, this would imply $M-1<L$, which is impossible.

The analogue of $(10.2),\left(10.2^{\prime}\right)$ for Case Bbb is $(L l+K k)\left(v_{r}-v_{1}\right)=$ $L\left(v_{r}-v_{r-1}\right)$. Then $L=1$ gives $r=2, L l+K k=L$, which entails $k=l-1$. By Property 5.5, $l=M m$, and the equality $L(l-1) v_{r}+L v_{r-1}=K k v_{1}$ gives the desired relation for $v_{1}, v_{2}$.

10.12 and 10.13 will be concerned with the cases $r=3$ and $r=2$ respectively. For these values of $r$, they lead to a complete description of the triples $(K, L, M),(k, l, m)$ and $\left(v_{i}\right)$ for which $\mathcal{P}_{\bar{\sigma}}$ has no singletons (see the final Remark in this section).

10.12. For $r=3$, we have $v_{3}=-v_{1}, v_{2}=0$ with the following exceptions (belonging to Case Bba): $K=-L, M=2 L, k=l-1, l=2 m, m \geq 2$, and

$$
v_{2}=-\frac{l-1}{3 l-1} v_{1}, v_{3}=-\frac{3 l-2}{3 l-1} v_{1} \quad \text { or } \quad v_{2}=\frac{l-1}{3 l-2} v_{1}, v_{3}=-\frac{3 l-1}{3 l-2} v_{1} .
$$


Proof. For Cases Ba, this is immediate by Properties 5.3 and 5.4. Cases Bbb, Bbc are impossible for $r>2$ by 10.11 .

In Case A, we have shown $v_{2}=0$ in 10.3 and $K k=L(l-1)$ (in particular, $l>1$ by 10.1). Assume that $v_{3}^{\prime}<2 v_{2}^{\prime}$ (equivalently, $v_{1}+v_{3}<0$ ). Then $\sigma(1, \ldots, 1)_{l}<\sigma(\ldots, 1,2)_{l}<\sigma(\ldots, 1,3)_{l}<\sigma(\ldots, 1,2,2)_{l}$ and no other point of $A_{l}$ is mapped to the closed interval spanned by these points. Similarly for $k$ and $m$ (of course, the last point exists only for $k>1$ resp. $m>1$ ). As before, we assume $\operatorname{gcd}(K, L, M)=1$. If $L=1$, then it follows from $K \geq 1, M \geq 2$ that $\bar{\sigma}(\ldots, 1,2)_{k}, \bar{\sigma}(\ldots, 1,2)_{m} \geq \bar{\sigma}(\ldots, 1,2,2)_{l}$, hence $\bar{\sigma}(\ldots, 1,3)_{l}$ would be isolated. If $L>1$, then by $10.6, M=L+K$ and $\bar{\sigma}(\ldots, 1,2)_{k}=\bar{\sigma}(\ldots, 1,2)_{m}$. Another consequence is $\operatorname{gcd}(K, L)=1$, which implies $L \mid k$, thus $k>1$. We get $\bar{\sigma}(\ldots, 1,3)_{k}<\bar{\sigma}(\ldots, 1,3)_{m}$, hence in order that neither $\bar{\sigma}(\ldots, 1,3)_{l}$ nor $\bar{\sigma}(\ldots, 1,3)_{k}$ are isolated, we must have $\bar{\sigma}(\ldots, 1,3)_{l}=\bar{\sigma}(\ldots, 1,3)_{k}$ (see also 10.7). This gives $L v_{3}^{\prime}=L v_{2}^{\prime}+K v_{3}^{\prime}$ and consequently, $M v_{3}^{\prime}=L v_{2}^{\prime}+2 K v_{3}^{\prime}$, which means that $\bar{\sigma}(\ldots, 1,3)_{m}=\bar{\sigma}(\ldots, 1,3,3)_{k}$. Another consequence is $K<L$, hence $\bar{\sigma}(\ldots, 1,2,2)_{k}<\bar{\sigma}(\ldots, 1,2,2)_{l}$, and it would follow that $\bar{\sigma}(\ldots, 1,2,2)$ is isolated. By symmetry, $v_{1}^{\prime \prime}<2 v_{2}^{\prime \prime}$ is impossible, which finishes Case A.

Finally, in Case Bba, subtracting the equations in Property 5.6 gives $K k=-L(l-1)$ (in particular, $l>1$ ), and then addition leads to $v_{2}=$ $-(l-1)\left(v_{1}+v_{3}\right)$. Again, we assume that $v_{3}^{\prime}<2 v_{2}^{\prime}$. Then $v_{2}^{\prime}>v_{3}^{\prime}-v_{2}^{\prime}=$ $v_{2}^{\prime \prime}$ and by $10.9, L=1$ (assuming $\operatorname{gcd}(K, L, M)=1$ ). Hence $\bar{\sigma}(1,3, \ldots)_{l}$, $\bar{\sigma}(\ldots, 1,2)_{k}<\bar{\sigma}(2,2,3, \ldots)_{l}$. It follows that $\bar{\sigma}(2,2,3, \ldots)_{l}=\bar{\sigma}(2,3, \ldots)_{m}$ and this gives $M=2$. Consequently, $\bar{\sigma}(\ldots, 1,2)_{m}=\bar{\sigma}(\ldots, 1,2,2)_{l}$, and (see the argument for Case A with $L=1$ ) it follows that $\bar{\sigma}(\ldots, 1,3)_{l}=$ $\bar{\sigma}(2,3, \ldots)_{k}$. This means $L v_{3}^{\prime}=L v_{2}^{\prime}+K v_{2}^{\prime \prime}$, hence $K=-1, k=l-1$, $l=2 m$. Since $m=1$ would give $k=1$, we have $m \geq 2, l \geq 4, k \geq 3$. Now we consider $(2,2,3, \ldots)_{k}$. We have $\bar{\sigma}(2,2,3, \ldots)_{k}-\bar{\sigma}(1, \ldots, 1)_{l}=v_{2}^{\prime}+2 v_{2}^{\prime \prime}$. As $M=2$ implies that $\bar{\sigma}\left(A_{m}\right) \subseteq \bar{\sigma}\left(A_{l}\right)$, we have $\bar{\sigma}(2,2,3, \ldots) \in \bar{\sigma}\left(A_{l}\right)$, and (using $v_{3}^{\prime}=v_{2}^{\prime}+v_{2}^{\prime \prime}$ ) this gives $v_{2}^{\prime}+2 v_{2}^{\prime \prime}=a_{1} v_{2}^{\prime}+a_{2} v_{2}^{\prime \prime}$ with $0 \leq a_{2} \leq a_{1}$. Clearly $a_{1} \geq 2$, and $v_{2}^{\prime \prime}<v_{2}^{\prime}$ implies $a_{1}<3$. The only possibility is $a_{1}=2$, $a_{2}=1$ (i.e. $\left.\bar{\sigma}(2,2,3, \ldots)_{k}=\bar{\sigma}(\ldots, 1,2,3)_{l}\right)$. It follows that $v_{2}^{\prime}=2 v_{2}^{\prime \prime}$, i.e. $2 v_{3}+v_{1}=3 v_{2}$. Combined with the formula for $v_{2}$ above, this gives the first solution for $v_{1}, v_{2}, v_{3}$. The second one is obtained by reflecting the sequence, it comes from the case $v_{3}^{\prime}>2 v_{2}^{\prime}$.

10.13. For $r=2$, Case $\mathrm{A}, v_{2}=-v_{1}, K>0$, there are two possibilities:

(i) $k=l-2, m \mid l, K=L, M=\frac{l}{m} L, l \geq 4$, where for $l=4$ only $m=1$ is possible,

(ii) $k=m-1, l=2 m, K=M=2 L, l \geq 4$.

Proof. This is similar to the first part of the proof of 10.12 . 
REMARK. The results show certain interdependencies among the cases. If the numbers $v_{i}$ lie symmetric with respect to 0 (i.e., $v_{r+1-i}=-v_{i}$ for all $i$, then $\sigma\left(r+1-i_{k}, \ldots, r+1-i_{1}\right)=-\sigma\left(i_{1}, \ldots, i_{k}\right)$, i.e. the sets $\sigma\left(A_{k}\right)$, $\sigma\left(A_{l}\right), \sigma\left(A_{m}\right)$ are also symmetric. Hence, if there is given a triple $(K, L, M)$, $\bar{\sigma}$ is defined by (4.1), (4.2) and the corresponding partition $\mathcal{P}_{\bar{\sigma}}$ has no singletons, then the same is true if we change the sign of one (or more) of the numbers $K, L, M$. If $(K, L, M)$ belongs to Case A, then (reordering $k, l, m$ correspondingly), the triple $(-K, L, M)$ leads to Case Bba (if $K>0$ ), $(-L, M, K)$ gives Case Bab, $(-M, L, K)$ Case Baa and conversely ("transference scheme").

For $r=2$, one can show that all triples described in 10.13 generate partitions without singletons. In Case Bba, it follows easily from Property 5.6 that $v_{2}=-v_{1}$, for Cases Ba this is contained in (5.3), and for Case A with $v_{2}>0$ see 10.3. Hence for $r=2$, the partitions of type B except Bbb, Bbc can all be derived from 10.13 and 10.1 using the transference scheme (where the limit case 10.1 leads to 10.8). Similarly (but not with the same $v_{i}$ ), the asymmetric case 10.2 of $\mathrm{A}$ is related to case 10.11 of $\mathrm{Bbb}$ (see also Section 13(a)). This gives (up to reorderings) all possibilities (for $r=2$ ) of partitions $\mathcal{P}_{\bar{\sigma}}$ without singletons.

For $r=3$, the situation is similar: apart from the exceptional case in 10.12 , we always have (by 10.12) $v_{3}=-v_{1}, v_{2}=0$. Case $\mathrm{A}$ is characterized by $K>0, M>L>0$ (Properties 5.2, 5.6), $L l=M m, K k=L(l-1)(10.3)$ and either $L \mid \operatorname{gcd}(K, M)$ or $M=K+L(10.6)$. All triples $(K, L, M)$ having these properties for some natural numbers $k, l, m$ (necessarily distinct) define partitions $\mathcal{P}_{\bar{\sigma}}$ without singletons. Type B (apart from the exception 10.12) can be derived from this by the transference scheme.

For $r \geq 4$, the situation becomes more complicated and a complete description of all possibilities seems difficult. An example for Case A with $v_{r} \neq-v_{1}$ (for $r=10$ ) can be found in [G, p. 80]. The transference scheme still works for the part of $\bar{\sigma}(A)$ near the extremal values: as pointed out before 10.9 , (10.2) carries over to the cases of type B; 10.9, 10.10 are the counterparts of 10.6. But for 10.7, the corresponding statement described in the Remark to 10.10 is not the immediate transcription.

11. Consequences of the equations from $\mathcal{P}$. Returning to the starting point in Section 2, let $\mathcal{P}$ be a partition of $A$ and write $\bar{S}(\mathcal{P})$ for the set of solutions $\mathbf{n}=(h, n, q)$ where (2.2) strengthens to $\sum_{\mathbf{i} \in I} p_{\mathbf{i}}(\mathbf{n}) \boldsymbol{\alpha}_{\mathbf{i}}^{\mathbf{n}}=0$ for all $I \in \mathcal{P}$. Note that this is slightly different from [L2] and [SS2], since $\bar{S}(\mathcal{P})$ gets larger when $\mathcal{P}$ is replaced by a more coarse partition $(\mathcal{P} \mapsto \mathcal{H}(\mathcal{P})$ has the opposite behaviour). As mentioned before, it follows from Laurent's results (see e.g. [SS2, Th. A]) that if $\bar{S}(\mathcal{P})$ is infinite, then there is a refinement $\mathcal{P}^{\prime}$ of $\mathcal{P}$ such that $\bar{S}\left(\mathcal{P}^{\prime}\right)$ is still infinite and $\mathcal{H}\left(\mathcal{P}^{\prime}\right)$ is non-trivial. Hence, we 
can assume that $\mathcal{H}(\mathcal{P})$ is non-trivial. By Section 3 (and Theorem 2) we can assume that $\mathcal{P}$ has no singletons and by the Proposition (Section 6 ), we must have $g=1$. Then (Section 4) there exists $\alpha$ and roots of unity $\zeta_{i}$ such that $\alpha_{i}=\alpha^{v_{i}} \zeta_{i}(i=1, \ldots, r)$. We can assume that $\zeta_{1}=1$.

Take a non-zero triple $(K, L, M) \in \mathcal{H}(\mathcal{P})$ and define $\bar{\sigma}$ by (4.2). Then $\mathcal{P}_{\bar{\sigma}}$ is coarser than $\mathcal{P}$ (or possibly equal), giving $\bar{S}(\mathcal{P}) \subseteq \bar{S}\left(\mathcal{P}_{\bar{\sigma}}\right)$. Hence, it will be enough to consider partitions $\mathcal{P}=\mathcal{P}_{\bar{\sigma}}$ without singletons, defined by some non-zero integer triple $(K, L, M)$. As a first step, we will study the equations coming from the extremal values of $\bar{\sigma}$ (using our knowledge from Sections 5 and 10). If $\bar{S}\left(\mathcal{P}_{\bar{\sigma}}\right)$ is infinite, we will deduce in all cases that $f_{1}, f_{2}, f_{r-1}, f_{r}$ are constant (see 11.3 and the Remark following it). This will give various equations for the polynomials $f_{i}$ in the description (2.1) of the recurrence sequence $\left(u_{n}\right)$ (recall that all $f_{i}$ are assumed to be non-zero). In most cases it will result that, possibly after replacing $\alpha_{i}$ by appropriate roots and translating the sequence $\left(u_{n}\right)$, we can always assume that $\bar{S}\left(\mathcal{P}_{\bar{\sigma}}\right) \subseteq$ $\mathbb{Q} \cdot(K, L, M)\left(\subseteq \mathbb{Q} \cdot \mathcal{H}\left(\mathcal{P}_{\bar{\sigma}}\right)\right)($ compare [L2, Th. $\left.1(\mathrm{i})]\right)$ : if we have a purely exponential equation - which means in our case that all the polynomials $f_{i}$ in (2.1) are constant - then one knows that $\bar{S}(\mathcal{P})$ is contained in the union of finitely many cosets of $\mathcal{H}(\mathcal{P})$, but still one has to show that the translating vector can be chosen with three equal coordinates). The exceptions are: Case A with $v_{r}=0$ (Property 10.2) or $K=0$ (Property 10.1), Case Ba with $M=0$ (Property 10.8), Case Bba with $r=3, v_{3} \neq-v_{1}$ (Property 10.12) and Cases Bbb, Bbc (Property 10.11). Hence, these exceptions occur for $r=2,3$ only.

11.1. Assume that $\left\{(1, \ldots, 1)_{l},(1, \ldots, 1)_{m}\right\} \in \mathcal{P}$ and that $\alpha_{1}$ is not a root of unity (i.e. $v_{1} \neq 0$ ). If $\bar{S}(\mathcal{P})$ is infinite, then $f_{1}$ is constant and the equation for this set becomes

$$
b f_{1}^{l} \alpha_{1}^{n l}+c f_{1}^{m} \alpha_{1}^{q m}=0 .
$$

If $f_{1}$ is constant, then $t=n l-q m$ has to be constant for $(h, n, q) \in \bar{S}(\mathcal{P})$.

Proof. Following the expansion leading to (2.2), the equation arising for this set is $b f_{1}(n)^{l} \alpha_{1}^{n l}+c f_{1}(q)^{m} \alpha_{1}^{q m}=0$. If $\bar{S}(\mathcal{P})$ is infinite, then (by arguments as in the last part of Section 3) it comprises infinitely many values $(n, q)$. Since $l \neq m$, it follows from [SS1, Lemma 3, p. 234] that $f_{1}$ has to be constant (in the description of the cases where equation (8.1) of [SS1] has infinitely many solutions one has that either $f$ or $g$ constant or, by (8.3), $f$ and $g$ of the same degree; neither possibility can occur in our situation). For $f_{1}$ constant, one obtains (11.1). Then $t$ has to be constant, unless $\alpha_{1}$ is a root of unity.

REMARK. To simplify the formulas, we can always assume that $t=0$ by the following argument: considering appropriate roots of the $\alpha_{i}$, the sequence 
$\left(u_{n}\right)_{n \in \mathbb{Z}}$ can be extended (using (2.1)) to rational indices (for the present purpose, $n \in \frac{1}{l-m} \cdot \mathbb{Z}$ is sufficient). Thus, we can define a new recurrence sequence $\left(u_{n}^{\prime}\right)_{n \in \mathbb{Z}}$, containing $\left(u_{n}\right)_{n \in \mathbb{Z}}$ as a subsequence, by $u_{n}^{\prime}=u_{(n+t) /(l-m)}$. Under this transformation, the condition $n l-q m=t$ for members of the sequence $\left(u_{n}\right)$ translates to $n l-q m=0$ for members of the sequence $\left(u_{n}^{\prime}\right)$.

11.2. Assume that $f_{1}, f_{r}$ are constant, the sets $\left\{(1, \ldots, 1)_{k},(\ldots, 1,2)_{l}\right\}$, $\left\{(r, \ldots, r)_{k},(r-1, r, \ldots)_{l}\right\}$ belong to $\mathcal{P}$ and $\bar{S}(\mathcal{P})$ is infinite. Then $f_{2}, f_{r-1}$ are constant and the equation for the first of the two sets becomes

$$
a f_{1}^{k} \alpha_{1}^{h k}+b l f_{1}^{l-1} f_{2}\left(\alpha_{1}^{l-1} \alpha_{2}\right)^{n}=0 .
$$

From the standard setting of Sections 5 and 10, we here need only that $v_{1}<v_{2}, v_{r-1}<v_{r}$ and $v_{1}<0 \leq v_{r}$.

Proof. Originally, we obtain: $a f_{1}^{k} \alpha_{1}^{h k}+b l f_{1}^{l-1} f_{2}(n)\left(\alpha_{1}^{l-1} \alpha_{2}\right)^{n}=0$ for the first set. Now, constancy of $f_{2}, f_{r-1}$ can be proved by considering this equation together with the other one (which is obtained by replacing the indices $(1,2)$ by $(r, r-1))$ and again examining the exceptional cases described in [SS1, Lemma 3, p. $234 \mathrm{f}]$. We include here a more direct argument. The first equation can be written as $a f_{1}^{k} \alpha^{e_{1}} \eta_{1}+b l f_{1}^{l-1} f_{2}(n)=0$ and the second as $a f_{r}^{k} \alpha^{e_{r}} \eta_{r}+b l f_{r}^{l-1} f_{r-1}(n)=0$, where $\eta_{1}, \eta_{r}$ are roots of unity (they can be assumed to be independent of $h, n), e_{1}\left(=e_{1}(h, n)\right)=(h k-n l) v_{1}+n\left(v_{1}-v_{2}\right)$, $e_{r}=(h k-n l) v_{r}+n\left(v_{r}-v_{r-1}\right)$. If e.g., $f_{2}$ is not constant, then $\left|f_{2}(n)\right| \rightarrow \infty$ and $\ln \left|f_{2}(n)\right| \ll \ln |n|$ for $|n| \rightarrow \infty$. It follows that $|\alpha| \neq 1,\left|e_{1}\right| \rightarrow \infty$ and $\left|e_{1}\right| \ll \ln \left|f_{2}(n)\right|$. If $f_{r-1}$ is also not constant, we get corresponding estimates for $\left|e_{r}\right|$. But observe that $v_{r} e_{1}-v_{1} e_{r}=n\left(v_{r}\left(v_{1}-v_{2}\right)-v_{1}\left(v_{r}-v_{r-1}\right)\right)$ and $v_{1}<0 \leq v_{r}$. This gives a contradiction. Similarly, if $f_{r-1}$ is constant.

11.3. Assume that the triple $(K, L, M)$ defining $\bar{\sigma}$ leads to Case $\mathrm{A}$ with $v_{r}>0, K>0$ and furthermore that $\bar{S}\left(\mathcal{P}_{\bar{\sigma}}\right)$ is infinite (in particular, $\mathcal{P}_{\bar{\sigma}}$ has no singletons $)$. Then $f_{1}, f_{2}, f_{r-1}, f_{r}$ are constant.

If $t=0$ in 11.1, then $\bar{S}\left(\mathcal{P}_{\bar{\sigma}}\right) \subseteq \mathbb{Z} \cdot(K, L, M)$.

As in Section 10, we here make the assumption $\operatorname{gcd}(K, L, M)=1$.

Proof. Recall the definition of Case A in Property 5.2. The set considered in 11.1 and its opposite counterpart belong to $\mathcal{P}_{\bar{\sigma}}$, it follows that $f_{1}, f_{r}$ are constant. By 11.2, $f_{2}, f_{r-1}$ are constant. From (11.1) and its opposite counterpart (replacing the index 1 by $r$ ) and the assumption $t=0$ (i.e., $n l=q m)$, it follows that $\left(f_{1} / f_{r}\right)^{l-m}=1$, i.e., $f_{1} / f_{r}$ is a root of unity. Now, we have to distinguish two subcases that came up in Section 10.

(a) Assume $L>1$. Then by Property $10.6, \bar{\sigma}(\ldots, 1,2)_{k}=\bar{\sigma}(\ldots, 1,2)_{m}$ and $\operatorname{gcd}(L, M)=1$. If $\bar{\sigma}(\ldots, 1,2)_{m} \in \bar{\sigma}\left(A_{l}\right)$, then $L \mid v_{2}^{\prime}$. Hence, if $p$ is any prime with $p \mid L$, we see from the Remark after 10.6 that $\left|\bar{\sigma}(\mathbf{i})-L l v_{1}\right|_{p}<$ 
$\left|M v_{2}^{\prime}\right|_{p}$ for all $\mathbf{i} \in A_{l}$, i.e., a contradiction. Consequently (see also Property 5.1), $\left\{(\ldots, 1,2)_{k},(\ldots, 1,2)_{m}\right\} \in \mathcal{P}_{\bar{\sigma}}$ and the corresponding equation gives (compare 11.4)

$$
a k f_{1}^{k}\left(\alpha_{1}^{k-1} \alpha_{2}\right)^{h}+c m f_{1}^{m}\left(\alpha_{1}^{m-1} \alpha_{2}\right)^{q}=0 .
$$

Combining with the opposite equation (and bearing in mind that $f_{r} / f_{1}$ is a root of unity), we find that $\left(\alpha_{r} / \alpha_{1}\right)^{h(k-1)-q(m-1)}\left(\alpha_{r-1} / \alpha_{2}\right)^{h-q}$ is a root of unity, leading to the equation $(h(k-1)-q(m-1))\left(v_{r}-v_{1}\right)+$ $(h-q)\left(v_{r-1}-v_{2}\right)=0$. Replacing on the left side $(h, q)$ by $(K, M)$ and rearranging gives the term $(K k-M m)\left(v_{r}-v_{1}\right)+(M-K)\left(v_{r-1}-v_{r}+v_{1}-v_{2}\right)$, and this is also zero by (10.2) and the identities $L l=M m$ and $M-K=L$. Now $t=0$ gives the equation $n l=q m$. Thus $(h, n, q)$ and $(K, L, M)$ are common solutions of two linear equations (that are clearly independent), hence $(h, n, q) \in \mathbb{Q} \cdot(K, L, M)$ (and in particular $q=h+n)$.

(b) Assume that $L=1, K>0$. Then by Property 10.4, we have $r=2$ or 3 . We describe the case $r=3$. By Property $10.3, K k=L(l-1)$, and by Property $10.12, v_{3}=-v_{1}$. Thus $\alpha_{1} \alpha_{3}$ is a root of unity. Using (11.2) and its opposite counterpart, it follows that $\left(\alpha_{1} / \alpha_{3}\right)^{h k-m(l-1)}$ is a root of unity. This gives $h k=n(l-1)$. Together with $n l=q m$ (from $t=0$ ), we have again two linear equations for $(h, n, q)$ that are also satisfied by $(K, L, M)$. The case $r=2$ is similar.

Remark. In Case A, $K=0$ implies $r=2$ (see 10.1), and constancy of $f_{1}, f_{2}$ follows from 11.1. In Case A with $v_{r}=0$, one has by $10.2, r=2$, $k=l-1, K=L=1 .\left\{(1,2, \ldots)_{k},(1,2, \ldots)_{l}\right\} \in \mathcal{P}_{\bar{\sigma}}$ (compare also Section 9(b)) gives another equality. Then constancy of $f_{2}$ can be shown as in 11.1.

Quite similarly, one can treat the cases of type B with the exceptions mentioned at the beginning of the section. We sketch the argument:

For Case Bba we can still apply 11.1 and its opposite counterpart. Equation (11.2) has to be replaced by $a f_{r}^{k} \alpha_{r}^{h k}+b f_{1}^{l-1} f_{2}(n)\left(\alpha_{1}^{l-1} \alpha_{2}\right)^{n}=0$ and its counterpart. This can be treated as in 11.2, giving constancy of $f_{2}, f_{r-1}$ (with $e_{1}=h k v_{r}-n l v_{1}+n\left(v_{1}-v_{2}\right), e_{r}=h k v_{1}-n l v_{r}+n\left(v_{r}-v_{r-1}\right)$, one has $\left.v_{1} e_{1}-v_{r} e_{r}=n l\left(v_{r}^{2}-v_{1}^{2}\right)+n\left(v_{1}\left(v_{1}-v_{2}\right)-v_{r}\left(v_{r}-v_{r-1}\right)\right)\right)$. Up to this point, the argument applies as well in the exceptional case of Property 10.12. Then, for $v_{r}=-v_{1}$, the remaining part can be done as in 11.3. Case Bbb (resp. Bbc) can be treated as Case A with $v_{r}=0$ above.

In Case Ba, the equation used in 11.1 has to be replaced by $a f_{r}(h)^{k} \alpha_{r}^{h k}+$ $b f_{1}(n)^{l} \alpha_{1}^{n l}=0$ and its opposite counterpart. A similar reasoning shows that $f_{1}, f_{r}$ have to be constant. Moreover, $t$ has to be replaced by $h k+n l$. Also the arguments of 11.2, 11.3 can be adapted to this case. E.g., in Case Baa with $M>0, L>1$, the analogues of (11.3) are $a k f_{r-1} f_{r}^{k-1}\left(\alpha_{r-1} \alpha_{r}^{k-1}\right)^{h}+$ $c m f_{1}^{m-1} f_{2}\left(\alpha_{1}^{m-1} \alpha_{2}\right)^{q}=0$ and its counterpart. If $h k+n l=0$, it follows that $f_{1} / f_{r}$ and $\left(\alpha_{r} / \alpha_{1}\right)^{h(k-1)+q(m-1)}\left(\alpha_{r-1} / \alpha_{2}\right)^{h+q}\left(f_{r-1} / f_{2}\right)^{2}$ are roots of unity. 
11.4. Keep the assumptions of 11.3 (including $t=0$ ) and assume in addition that $\left\{(\ldots, 1, i)_{k},(\ldots, 1, i)_{l}\right\} \in \mathcal{P}_{\bar{\sigma}}$ for some $i$. Then $f_{i}$ is a monomial and the equation for the set becomes (with $s=\operatorname{deg} f_{i}$ )

$$
a k f_{1}^{k} K^{s} \zeta_{i}^{h}+b l f_{1}^{l} L^{s} \zeta_{i}^{n}=0 .
$$

Proof. Originally, the equation for the set is

$$
a k f_{1}^{k-1} f_{i}(h)\left(\alpha_{1}^{k-1} \alpha_{i}\right)^{n}+b l f_{1}^{l-1} f_{i}(n)\left(\alpha_{1}^{l-1} \alpha_{i}\right)^{n}=0 .
$$

From 11.3, we deduce that $n=\frac{L}{K} h$ and since the assumption gives $L v_{2}^{\prime}=$ $(L-K) v_{i}^{\prime}$, we conclude that $f_{i}\left(\frac{L}{K} h\right) / f_{i}(h)$ has to be constant. Then $f_{i}$ must be a monomial and (11.4) follows.

REMARK. Similar statements hold in the other cases described in the Remark to Property 10.10. E.g., in Case Bab with $K<-1$, if $\left\{(\ldots, 1, i)_{k}\right.$, $\left.(j, r, \ldots)_{m}\right\},\left\{(j, r, \ldots)_{k},(\ldots, 1, i)_{m}\right\} \in \mathcal{P}_{\bar{\sigma}}$, then $f_{i}, f_{j}$ are monomials of the same degree and one obtains analogues of the last formula in the Remark to 11.3 (with $(r-1,2)$ replaced by $(j, i)$ and additional factors $K^{s}, M^{s}$ similar to $(11.4))$.

12. Polynomial identities. The proof of Theorem 1 will lead to polynomial identities of the type

$$
x^{e} p_{1}\left(x^{K}\right)^{k}+p_{2}\left(x^{L}\right)^{l}+p_{3}\left(x^{M}\right)^{m}=0
$$

where $p_{1}, p_{2}, p_{3}$ are three non-zero polynomials, and we will use the method of Mason ([M1, Lemma 2, p. 14], see also [M2]) to show that in most of our applications (12.1) has no solutions.

Lemma 1. Let $\varphi_{1}, \varphi_{2}, \varphi_{3}$ be coprime complex polynomials and assume that $\varphi_{1}+\varphi_{2}+\varphi_{3}=0$. Let $\nu$ be the cardinality of the set of zeros of $\varphi_{1} \varphi_{2} \varphi_{3}$ (i.e., the number of zeros counted without multiplicities). Then $\operatorname{deg} \varphi_{i} \leq$ $\nu-1$ for $i=1,2,3$. If equality holds for some $i$, then $\varphi_{1}^{\prime} \varphi_{2}-\varphi_{2}^{\prime} \varphi_{1}$ divides $\varphi_{1} \varphi_{2} \varphi_{3}$.

Proof. The inequality is a special case of [M1, Lemma 2, p. 14]. The idea is that multiple zeros of $\varphi_{1} \varphi_{2} \varphi_{3}$ give zeros of $\varphi_{1}^{\prime} \varphi_{2}-\varphi_{2}^{\prime} \varphi_{1}$ with multiplicity reduced by 1 . In the case of equality, this exhausts all zeros of $\varphi_{1}^{\prime} \varphi_{2}-\varphi_{2}^{\prime} \varphi_{1}$.

In our case, the terms of the sum in (12.1) are not necessarily coprime, i.e., there may be common zeros, but the (rather technical) Lemma 2 shows that, due to the relations found in Sections 5 and 10, the occurrences are rather limited.

We take up the notations of Section 5. $v_{1}<\cdots<v_{r}$ are assumed to be rationals $(r \geq 2)$. $K, L, M$ denotes integers with $\operatorname{gcd}(K, L, M)=1$, belonging to Case A (Property 5.2) without singletons and with $v_{r}>0$. In 
fact, from the properties of partitions without singletons only the statements (5.1), (10.2) and Property 10.6 are used in the following proof. Apart from the bound on the degree, there are no restrictions on the polynomials $p_{1}, p_{2}, p_{3}$ (i.e., the non-zero coefficients need not be located at the powers $v_{1}^{\prime}, \ldots, v_{r}^{\prime}$, as in the construction of Section 13(a) for Case A). This additional generality will be needed when transferring the cases of type $\mathrm{B}$ to Case A.

LEMMA 2. In the above setting, assume that $v_{2}-v_{1}=v_{r}-v_{r-1}=1$ and $p_{1}, p_{2}, p_{3}$ are non-zero complex polynomials such that $\operatorname{deg} p_{i} \leq v_{r}-v_{1}$ for $i=1,2,3$ and (12.1) holds with $e=L$.

If $\xi \neq 0$ defines a common zero with multiplicity $\nu_{i}$ in $p_{i}$, then only the following cases are possible for $L>1$ :

( $\alpha) \nu_{2}=L, \nu_{3}=M, L l-K k=1, L>K, \nu_{1} \geq K+1$.

( $\beta) \nu_{1}=l / l_{0}, \nu_{2}=k / k_{0}$, where $l_{0}=\operatorname{gcd}(k, l)=L l-K k$ and furthermore $l=M, m=L>K, \nu_{3} \geq(k+l) / l_{0}$.

$(\gamma) \nu_{1}=m / l_{1}, \nu_{3}=k / l_{1}$, where $l_{1}=\operatorname{gcd}(k, m)=L l-K k$ and furthermore $l=M, m=L \leq K+1, \nu_{2} \geq(k-m) / l_{1}+1$.

For $L=1$, we have necessarily $r=3$ and the three cases:

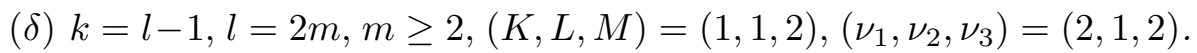

(ع) $k=1, m=2, \nu_{1}=2$.

( $\varphi) m=1, k=2, \nu_{3}=2$.

Proof. The assumption " $\xi$ defines a common zero" refers to the corresponding powers, i.e., $p_{1}\left(\xi^{K}\right)=p_{2}\left(\xi^{L}\right)=p_{3}\left(\xi^{M}\right)=0$ (with the given multiplicities). Then $\xi$ has multiplicity $k \nu_{1}$ in $p_{1}\left(x^{K}\right)^{k}$, and (12.1) entails that at least two of the numbers $\nu_{1} k, \nu_{2} l, \nu_{3} m$ have to be equal and the third one is not smaller than the other two.

For $r=2$, our assumption gives $\operatorname{deg} p_{i}=1$ and since $k, l, m$ are different, there can be no common zero $\xi \neq 0$. Hence $r \geq 3$ and we will discuss the following cases:

$$
\left(\alpha_{0}\right) \nu_{2} l=\nu_{3} m \leq \nu_{1} k, \quad\left(\beta_{0}\right) \nu_{1} k=\nu_{2} l \leq \nu_{3} m, \quad\left(\gamma_{0}\right) \nu_{1} k=\nu_{3} m \leq \nu_{2} l .
$$

The assumptions on $\left(v_{i}\right)$ give by (10.2):

$$
v_{r}-v_{1}=\frac{2 L}{L l-K k} .
$$

First assume that $L>1$. In case $\left(\alpha_{0}\right)$ we use (5.1): $L l=M m$ and Property 10.6, $\operatorname{giving} \operatorname{gcd}(L, M)=1$. Put $\nu_{0}=\operatorname{gcd}\left(\nu_{1}, \nu_{2}\right)$; it follows that $\nu_{2}=L \nu_{0}, \nu_{3}=M \nu_{0}$. Hence

$$
M \nu_{0} \leq v_{r}-v_{1}=\frac{2 L}{L l-K k} \leq 2 L
$$

and since $M>L$, we get $\nu_{0}=L l-K k=1$ and $M \leq 2 L$. By Property 10.6 
(and using $\operatorname{gcd}(K, L)=1$ ), it follows that $K<L$, and $1+K k=L l=\nu_{2} l \leq$ $\nu_{1} k$ gives $\nu_{1} \geq K+1$, proving all assertions of $(\alpha)$.

In case $\left(\beta_{0}\right)$, we have clearly $l \mid \nu_{1} l_{0}$, furthermore $L l-K k \geq l_{0}$ and (again using $L l=M m, \operatorname{gcd}(L, M)=1) M \mid l$. This gives

$$
1 \leq \frac{l}{M} \leq \frac{\nu_{1} l_{0}}{M} \leq \frac{\left(v_{r}-v_{1}\right) l_{0}}{M}=\frac{2 L}{M} \frac{l_{0}}{L l-K k} \leq \frac{2 L}{M}<2 ;
$$

it follows that $l=M=\nu_{1} l_{0} \leq 2 L$ and $L l-K k=l_{0}$. This entails that $\nu_{2}=k / l_{0}, m=L$ and (as in $\left.\left(\alpha_{0}\right)\right) K<L$. Furthermore,

$$
\nu_{3} \geq \nu_{2} \frac{l}{m}=\frac{k l}{l_{0} m}=\frac{k}{l_{0}} \frac{M}{L}=\frac{k}{l_{0}}\left(1+\frac{K}{L}\right),
$$

and $l / l_{0}-K k /\left(L l_{0}\right)=1 / L<1$ gives $\nu_{3} \geq(k+l) / l_{0}$, proving the assertions of $(\beta)$.

Similarly in case $\left(\gamma_{0}\right)$,

$$
1 \leq \frac{m}{L} \leq \frac{\nu_{1} l_{1}}{L} \leq \frac{\left(v_{r}-v_{1}\right) l_{1}}{L} \leq 2 .
$$

Recall that (Remark after Property 10.6) $k>m$. This excludes $\nu_{1} l_{1}=2 L$ and $L l-K k=2 l_{1}$ (since both would imply $\nu_{1}=v_{r}-v_{1}$, hence $\nu_{3}=\nu_{1} k / m>$ $\left.v_{r}-v_{1}\right)$. Thus, $\nu_{1} l_{1}=L$ and $L l-K k=l_{1}, v_{r}-v_{1}=2 \nu_{1}$, which entails $m=L$. Consequently, $l=M$ and $k=\nu_{3} l_{1}$. Since $\nu_{3} \leq v_{r}-v_{1}=2 \nu_{1}=2 L / l_{1}$, we get $k \leq 2 L=2 m$ and $l_{1}=L l-K k=M m-K k \geq(M-2 K) m=(L-K) m$. Now, $m \geq l_{1}$ gives $L-K \leq 1$. Furthermore,

$$
\nu_{2} \geq \nu_{3} \frac{m}{l}=\frac{k m}{l_{1} l}=\frac{k}{l_{1}} \frac{L}{M}=\frac{k}{l_{1}}\left(1-\frac{K}{M}\right)
$$

and $L / l_{1}-K k /\left(M l_{1}\right)=1 / M<1$ gives $\nu_{2} \geq(k-m) / l_{1}+1$ and proves all assertions of $(\gamma)$.

For $L=1$, recall that by (10.2), $r \leq 3$. Hence $r=3$, we have $\nu_{i} \leq 2$ $\left(=v_{r}-v_{1}\right)$ and by (10.2), $K k=l-1$. If $\nu_{2} l \leq \nu_{1} k, \nu_{3} m$, then (using $l>m$, $k \leq K k=l-1)\left(\nu_{1}, \nu_{2}, \nu_{3}\right)=(2,1,2)$. Then case $\left(\beta_{0}\right)$ would imply $k \mid l$, hence $k=1, l=2$, which is impossible. Case $\left(\alpha_{0}\right)$ leads to $(\delta)$. Finally, in case $\left(\gamma_{0}\right)$, we have $\nu_{3} m=\nu_{1} k \leq \nu_{2} l$. If $m>k$, then $\nu_{1}=2, \nu_{3}=1$, giving $m=2 k$. In combination with $K k=l-1, M m=l$, we get $2 M k=l=K k+1$, hence $(2 M-K) k=1$, giving $k=1, m=2$, i.e., case $(\varepsilon)$. Similarly, $m<k$ results in $\nu_{1}=1, \nu_{3}=2,2 m=k,(M-2 K) m=1$, giving $m=1, k=2$, i.e., case $(\varphi)$.

COROLlary. There can be at most one common zero $\neq 0$ of the three polynomials.

Proof. In $(\alpha)-(\gamma)$ we have $\nu_{3}>\left(\operatorname{deg} p_{3}\right) / 2$ (recall that we have $v_{r}-v_{1}=$ $2 L /(L l-K k)$; in $(\gamma)$ use $k>m)$ and the claim follows easily. Similarly in $(\delta)-(\varphi)\left(\right.$ since $\left.\operatorname{deg} p_{i} \leq 2\right)$. 
13. Proof of Theorem 1 (final). The main steps will be as follows: in (a) we show that the solutions $(h, n, q) \in \bar{S}\left(\mathcal{P}_{\bar{\sigma}}\right)$ correspond to certain identities of rational functions, resp. polynomial identities of the type (12.1). Then in (b) we consider the case where $v_{2}^{\prime}$ does not divide all $v_{i}^{\prime}$. Here we can use the equations from Section 11 to get a contradiction. In (c) we apply Mason's method and it turns out that in most of our cases where $v_{2}^{\prime}=v_{r-1}^{\prime \prime}=1$ the polynomial identity (12.1) has no solutions. The method leaves open several cases where one of the exponents gets small, in particular all cases where $k=1$ or $m=1$. These remaining cases are discussed in (d) $-(\mathrm{g})$.

(a) The state of the affair is as follows (see the introduction to Section 11): We assume that we have given an equation (1.1) with infinitely many solutions. Based on Laurent's theorem, it is enough to consider nonzero integer triples $(K, L, M)$ for which $\bar{S}\left(\mathcal{P}_{\bar{\sigma}}\right)$ is infinite and the partition $\mathcal{P}_{\bar{\sigma}}$ has no singletons. Furthermore, we are only left with the case $g=1$, i.e., $\alpha_{i}=\alpha^{v_{i}} \zeta_{i}$, where $v_{i} \in \mathbb{Z}, \zeta_{i}$ are roots of unity $(i=1, \ldots, r), \zeta_{1}=1$. Recall (2.1): $u_{n}=\sum_{i=1}^{r} f_{i}(n) \alpha_{i}^{n}$ and the notations $v_{i}^{\prime}=v_{i}-v_{1}, v_{i}^{\prime \prime}=v_{r}-v_{i}$. Take $(h, n, q) \in \bar{S}\left(\mathcal{P}_{\bar{\sigma}}\right)$, fix an integer triple $\left(K_{0}, L_{0}, M_{0}\right)$ defining $\mathcal{P}_{\bar{\sigma}}$ and put $\left(h_{0}, n_{0}, q_{0}\right)=(h, n, q)-\left(K_{0}, L_{0}, M_{0}\right)$. Now we consider the following rational functions:

$$
\begin{gathered}
\widetilde{p}_{1}(x)=\sum_{i=1}^{r} f_{i}(h) \zeta_{i}^{h} \alpha^{h_{0} v_{i}} x^{v_{i}}, \quad \widetilde{p}_{2}(x)=\sum_{i=1}^{r} f_{i}(n) \zeta_{i}^{n} \alpha^{n_{0} v_{i}} x^{v_{i}} \\
\widetilde{p}_{3}(x)=\sum_{i=1}^{r} f_{i}(q) \zeta_{i}^{q} \alpha^{q_{0} v_{i}} x^{v_{i}}
\end{gathered}
$$

Clearly, $\widetilde{p}_{1}\left(\alpha^{K_{0}}\right)=u_{h}, \widetilde{p}_{2}\left(\alpha^{L_{0}}\right)=u_{n}, \widetilde{p}_{3}\left(\alpha^{M_{0}}\right)=u_{q}$. Put

$$
\widetilde{p}_{0}(x)=a \widetilde{p}_{1}\left(x^{K_{0}}\right)^{k}+b \widetilde{p}_{2}\left(x^{L_{0}}\right)^{l}+c \widetilde{p}_{3}\left(x^{M_{0}}\right)^{m} .
$$

As in Section 2, the power expression $\widetilde{p}_{1}\left(x^{K_{0}}\right)^{k}$ can be expanded into a sum indexed by $A_{k}$, and for $\mathbf{i} \in A_{k}, \bar{\sigma}_{0}(\mathbf{i})$ gives the exponent of $x$ in the corresponding term of the sum $\left(\bar{\sigma}_{0}\right.$ being defined by (4.2) with $\left(K_{0}, L_{0}, M_{0}\right)$; by assumption $\mathcal{P}_{\bar{\sigma}_{0}}=\mathcal{P}_{\bar{\sigma}}$ ). Hence, the sets in $\mathcal{P}_{\bar{\sigma}}$ collect those terms of the sum containing the same power of $x$. If we substitute $x=\alpha$, this expansion of $a u_{h}^{k}=a \widetilde{p}_{1}\left(\alpha^{K_{0}}\right)^{k}$ gives (termwise) just the corresponding part of the sum (2.2). Thus $(h, n, q) \in \bar{S}\left(\mathcal{P}_{\bar{\sigma}}\right)$ means that in the expansion of $\widetilde{p}_{0}(x)$ those terms where the exponent of $x$ has any common value sum up to zero (formally: if $I=\left\{\mathbf{i} \in A: \bar{\sigma}_{0}(\mathbf{i})=j\right\}$ is any member of $\mathcal{P}_{\bar{\sigma}}, \mathbf{n}=(h, n, q)$, then $\alpha^{-j} \sum_{\mathbf{i} \in I} p_{\mathbf{i}}(\mathbf{n}) \alpha_{\mathbf{i}}^{\mathbf{n}}$ gives the coefficient of $x^{j}$ in $\left.\widetilde{p}_{0}(x)\right)$. Hence, $\widetilde{p}_{0}(x)=0$ and this leads to the identity of rational functions

$$
a \widetilde{p}_{1}\left(x^{K}\right)^{k}+b \widetilde{p}_{2}\left(x^{L}\right)^{l}+c \widetilde{p}_{3}\left(x^{M}\right)^{m}=0 .
$$

This has been shown for $\left(K_{0}, L_{0}, M_{0}\right)$ in place of $(K, L, M)$. But if an iden- 
tity (13.2) holds, it remains true if we multiply $(K, L, M)$ by a common factor. Hence, we can assume that in $(13.2) \operatorname{gcd}(K, L, M)=1$ (retaining the assumption used in most of Section 10). Conversely (same argument as above), (13.2) with (13.1) clearly implies that $(h, n, q) \in \bar{S}\left(\mathcal{P}_{\bar{\sigma}}\right)$.

For $K, L, M \geq 0$ (i.e., Case A) we put $p_{1}(x)=a^{1 / k} \widetilde{p}_{1}(x) x^{-v_{1}}, \quad p_{2}(x)=b^{1 / l} \widetilde{p}_{2}(x) x^{-v_{1}}, \quad p_{3}(x)=c^{1 / m} \widetilde{p}_{3}(x) x^{-v_{1}}$. Then $p_{1}, p_{2}, p_{3}$ are polynomials and by (5.1), (5.2), they satisfy the identity (12.1) with $e=L v_{2}^{\prime}$.

Similarly in the other cases, where $K<0, L, M \geq 0$. For example, in Case Bab, we put $p_{1}(x)=c^{1 / m} \widetilde{p}_{3}(x) x^{-v_{1}}, \quad p_{2}(x)=a^{1 / k} \widetilde{p}_{1}\left(\frac{1}{x}\right) x^{v_{r}}, \quad p_{3}(x)=b^{1 / l} \widetilde{p}_{2}(x) x^{-v_{1}}$ and obtain $(12.1)$ with $(K, L, M)($ resp. $(k, l, m))$ replaced by $\left(K^{\prime}, L^{\prime}, M^{\prime}\right)=$ $(M,-K, L)\left(\operatorname{resp} .\left(k^{\prime}, l^{\prime}, m^{\prime}\right)=(m, k, l)\right)$ and $e=(-K) v_{r-1}^{\prime \prime}$ (this is another aspect of the transference scheme between the cases, described at the end of Section 10).

Note that if $(h, n, q) \in \mathbb{Z} \cdot(K, L, M)$, it is possible to start the construction with $\left(K_{0}, L_{0}, M_{0}\right)=(h, n, q)$. Then (13.1) holds with $\left(h_{0}, n_{0}, q_{0}\right)=(0,0,0)$.

(b) Assume that $(K, L, M)$ leads to Case A and that $v_{2}^{\prime} \neq \operatorname{gcd}\left(v_{2}^{\prime}, \ldots, v_{r}^{\prime}\right)$. By Property 10.1, we have $K>0$, by Property 10.2, $v_{r}>0$, and by Properties 10.4, 10.12 (since clearly $r \geq 3$ ), we have $L>1$. By the Remark to 11.1 , we can assume $t=0$. Then 11.3 gives $(h, n, q) \in \mathbb{Z} \cdot(K, L, M)$. Computing the determinant of the coefficients of $a, b, c$ in equations (11.1)(11.3) gives $m f_{1}-k l f_{2}=0$. On the other hand, (11.2), (11.4) lead to $f_{1} \zeta_{i}^{n} L^{s}-k f_{2} \zeta_{2}^{n} \zeta_{i}^{h} K^{s}=0$ (using the fact that $h k v_{1}=n\left((l-1) v_{1}+v_{2}\right)$ by (5.2)). Combining these, we get $m K^{s} \zeta_{2}^{n} \zeta_{i}^{h}=l L^{s} \zeta_{i}^{n}$, and taking absolute values, this would imply $m K^{s}=l L^{s}$, hence (by (5.1)) $L K^{s}=M L^{s}$. But Property 10.6 gives $\operatorname{gcd}(M, K)=\operatorname{gcd}(M, L)=1$ and this is impossible (since $M>1$ ).

By the Remark at the end of Section 11, the same argument applies in the cases of type B (if $v_{2}^{\prime} \neq \operatorname{gcd}\left(v_{2}^{\prime}, \ldots, v_{n}^{\prime}\right)$ ) with the exception of the asymmetric case described in Property 10.12, where we cannot guarantee $(h, n, q) \in \mathbb{Z} \cdot(K, L, M)$. This exception is discussed in (e) and this will settle all cases where $v_{2}^{\prime} \neq \operatorname{gcd}\left(v_{2}^{\prime}, \ldots, v_{r}^{\prime}\right)$.

(c) We continue the investigation of (12.1), (13.2). By (b), we can assume that $v_{2}^{\prime} \mid v_{i}^{\prime}$ for all $i$ and the same for $v_{r-1}^{\prime \prime}$ (by reflecting the $\alpha_{i}$ ). The exponents appearing in the polynomials $p_{1}, p_{2}, p_{3}$ are $v_{i}^{\prime}$ or $v_{i}^{\prime \prime}$, hence we can divide them by $\operatorname{gcd}\left(v_{2}^{\prime}, \ldots, v_{r}^{\prime}\right)$ and assume that $\operatorname{gcd}\left(v_{2}^{\prime}, \ldots, v_{r}^{\prime}\right)=$ $\operatorname{gcd}\left(v_{1}^{\prime \prime}, \ldots, v_{r-1}^{\prime \prime}\right)=1$ (of course, this can make some $v_{i}$ non-integral). It follows that $v_{2}^{\prime}=v_{r-1}^{\prime \prime}=1$. 
We want to apply Lemma 1 of Section 12 . We start with Case A. First assume that the parts of the sum in (12.1) have no common zeros. By Section $11, f_{1}, f_{r}$ are (non-zero) constants and clearly $L>0$. Hence $\operatorname{deg}\left(p_{2}\left(x^{L}\right)^{l}\right)=$ $L l v_{r}^{\prime}=M m v_{r}^{\prime}$ and $p_{i}(0) \neq 0$. The cardinality of the set of zeros of the three parts is bounded by $\bar{\nu}=(K+L+M) v_{r}^{\prime}+1$. If $L>1$, then by Property 10.6, $M=L+K$, hence $\bar{\nu}=2 M v_{r}^{\prime}+1$. For $m>2$, Lemma 1 gives a contradiction; $m=2$ (a limiting case) is discussed in (d). For $L=1$, Lemma 1 gives (assuming still that there are no common zeros) $l v_{r}^{\prime} \leq(K+M+1) v_{r}^{\prime}$. By Property 10.4 we have $r \leq 3$, hence by Property $10.3, K k=l-1$ for $r=3$ and $K k=l-2$ for $r=2, v_{r}>0$. Then (recall that by (5.1) $l=M m$ ) elementary estimates show that the only possibilities are $(k, l, m)=(3,4,2)$ or $k=1$ or $m=1$. The first alternative is postponed to (e), the other two will be discussed in (f) for $r=3$ and in (g) for $r=2$.

Assume now that $L>1$ and that common zeros appear. Then we have the possibilities $(\alpha),(\beta),(\gamma)$ of Lemma 2. By the Corollary to Lemma 2, there can be only one common zero $\xi$. In case $(\alpha)$, cancellation of the common factor $(x-\xi)^{L l}$ reduces the degree of $p_{2}\left(x^{L}\right)$ by $L l=M m$. The bound $\bar{\nu}$ was based on simple zeros, and $\zeta \xi$, where $\zeta^{L}=1$, gives further zeros of $p_{2}\left(x^{L}\right)$ with multiplicity $\nu_{2}=L$ (similarly for $\left.p_{1}, p_{3}\right)$. Thus $\bar{\nu}$ can be reduced by $\left(\nu_{1}-1\right) K+\left(\nu_{2}-1\right) L+\left(\nu_{3}-1\right) M \geq K^{2}+(L-1) L+(M-1) M \geq M^{2}$. Then Lemma 1 gives $M m v_{r}^{\prime}-M m \leq 2 M v_{r}^{\prime}-M^{2}$, equivalently $(m-2) v_{r}^{\prime} \leq m-M$. For $m \leq M$, we get $m \leq 2$ and since $M>L, m \geq L \geq 2$, this is impossible. $m>M$ is also impossible, since $v_{r}^{\prime}=2 L \geq 4$ in case $(\alpha)$. In the alternatives $(\beta),(\gamma)$ the reduction of $\bar{\nu}$ is bigger than the reduction of the degree, hence this is also impossible.

Similarly, for $L=1, v_{r}>0$, case $(\delta)$ gives $l \leq 3$ in Lemma 1 , which is impossible. $(\varepsilon),(\varphi)$ are covered by the discussion in (f).

Note that if $p_{1}, p_{2}$ or $p_{3}$ have multiple zeros, then $m=2$ is also covered by Lemma 1 , since in this case the bound $\bar{\nu}$ can be reduced as well.

As observed in (a), the cases of type B can be transferred, leading also to a polynomial identity (12.1). In some asymmetric cases, the partition defined by $\left(K^{\prime}, L^{\prime}, M^{\prime}\right)$ may have singletons (see the Remark at the end of Section 10; for $r=2,3$ the correspondence is almost complete). But the properties needed in Lemma 2 remain true: (5.1) for $\left(K^{\prime}, L^{\prime}, M^{\prime}\right)$ results from (5.3) and Property 5.5, similarly for (10.2) (exclulding Case Bbb, resp. Bbc) and Property 10.6 (see again the end of Section 10). This is enough to be able to apply Lemma 2 to $\left(K^{\prime}, L^{\prime}, M^{\prime}\right)$ and the polynomials obtained from $\widetilde{p}_{i}$ as explained above. Hence, the argument above covers the cases of type B as well, with the exception of Case Bbb (resp. Bbc) where transference leads to Case A with $v_{r}=0$.

Case A with $v_{r}=0$ and Case Bbb (resp. Bbc) will be discussed in (g). 
(d) We now examine the limiting case $m=2, L>1$ that arose in (c). Since $L \leq m$, this gives $L=2, l=M=K+2$. First we want to show that this allows only the following three possibilities for $(k, l, m)$ : $(5,3,2),(3,5,2),(4,3,2)$. For $r>3$, it follows in Case A from Property 10.4 that $L l-K k=1$. This implies $K(k-2)=3$, hence $k-2 \in\{1,3\}$, giving the first two triples above. For $r=3$, by Property 10.3 we have $K k=L(l-1)$, which implies $K(k-2)=2$. As $K=2$ is impossible $($ since $\operatorname{gcd}(K, L)=1)$, we get $k=4$, giving the third triple. By a similar argument, $r=2$ would give $k=L=2$, which is impossible. The cases of type $\mathrm{B}$ can be transferred to Case A as before (recall that Properties 10.3 and 10.4 are direct consequences of (10.2)).

The arguments in the three cases are similar, we present them only in the first one, i.e. for $(k, l, m)=(5,3,2)$, which means $(K, L, M)=(1,2,3)$, $v_{r}^{\prime}\left(=\frac{2 L}{L l-K k}\right)=4$. Thus (12.1) becomes $x^{2} p_{1}(x)^{5}+p_{2}\left(x^{2}\right)^{3}+p_{3}\left(x^{3}\right)^{2}=0$ with $\operatorname{deg} p_{i}=4$.

By Section 11, $f_{1}, f_{2}, f_{r-1}, f_{r}$ are (non-zero) constants. By (13.1) and the definition of $p_{i}$ in (a), $p_{i}(0)$ is a non-zero multiple of $f_{1}$ or $f_{r}$, hence $p_{i}(0) \neq 0$. As noticed in (c), we can assume that the $p_{i}$ have only simple zeros and that there are no common zeros in the parts of (12.1). Then the case of equality in Lemma 1 gives (after cancellation)

$$
\left(2 p_{1}(x)+5 x p_{1}^{\prime}(x)\right) p_{2}\left(x^{2}\right)-6 x p_{2}^{\prime}\left(x^{2}\right) p_{1}(x)=c_{0} p_{3}\left(x^{3}\right)
$$

for some constant $c_{0}$ (see also the proof of Lemma 1). Comparing the coefficients of $x$ (and using $p_{2}(0) \neq 0$ ) gives $p_{1}^{\prime}(0)=0$, which is impossible, since $f_{2}, f_{r-1}$ are non-zero (recall that $v_{2}^{\prime}=v_{r-1}^{\prime \prime}=1$ ).

(e) In the first two cases of Property 10.12 we get (after reducing the powers) the polynomial identity $x^{2} p_{1}(x)^{l-1}+p_{2}(x)^{l}+p_{3}\left(x^{2}\right)^{l / 2}=0$ with $\operatorname{deg} p_{i}=3$, where $l=2 m \geq 4$ is even. Again, Lemma 1 excludes $l>4$ and multiple zeros of $p_{i}$. Considering the cases as in the proof of Lemma 2, the only possibility for common zeros is $\nu_{1}=\nu_{3}=2, \nu_{2}=1$ where a similar argument as in (c) applies. The limiting case $l=4$ leads to an identity $\left(2 p_{1}(x)+3 x p_{1}^{\prime}(x)\right) p_{2}(x)-4 x p_{1}(x) p_{2}^{\prime}(x)=c_{0} p_{3}\left(x^{2}\right)$, which can be handled as in (d) (observe that due to the special form of $v_{i}^{\prime}$ in 10.12 , we have $p_{2}^{\prime}(0)=0$ and we should have $\left.p_{1}^{\prime}(0) \neq 0\right)$. As remarked in 10.12 the second of the cases there can be reduced to the first one by reflection.

Consider now the remaining case $(k, l, m)=(3,4,2), L=1$ from (c) (excluding Case A with $v_{r}=0$ and Case Bbb). Then $r=3$ and the identity (12.1) becomes $x p_{1}(x)^{3}+p_{2}(x)^{4}+p_{3}\left(x^{2}\right)^{2}=0$ with $\operatorname{deg} p_{i}=2$. This is again a limiting case for Lemma 1, but unfortunately here the conclusion from Lemma 1 is not sufficient to give decisive information. By examining the coefficients of the powers of $x$ (in a similar way to (f), (g) below), one can again derive a contradiction. 
(f) We are left with the cases where $L=1, v_{2}^{\prime}=v_{r-1}^{\prime \prime}=1$ and either $m=1$ or $k=1$. By Property 10.4, we have $r \leq 3$. We now discuss $r=3$. We have $M m=l$ and by Property $10.3, K k=l-1$. Thus, for $m=1$, (12.1) becomes $x p_{1}\left(x^{K}\right)^{(l-1) / K}+p_{2}(x)^{l}+p_{3}\left(x^{l}\right)=0$. We have $l \geq 3$ and $\operatorname{deg} p_{i}=v_{r}^{\prime}=2$, i.e., $p_{i}(x)=f_{i 0}+f_{i 1} x+f_{i 2} x^{2}(i=1,2,3)$. Here, Lemma 1 gives only few restrictions (and at least for $l=3$, the polynomial identity has non-zero solutions). We compare the coefficients of the polynomials arising above. For $K \geq 2$ (which implies $l \geq 5$ ) the equation coming from $x^{2}$ and that from $x^{3}$ or $x^{4}$ (for $K=2$ ) have only non-integer common solutions $l$. Hence we are left with $K=1$.

Now, we have to make use of the special form of the coefficients appearing in $p_{i}$. By Section 11, we know that the polynomials $f_{1}, f_{2}, f_{3}$ are constant and that we can assume $\bar{S}\left(\mathcal{P}_{0}\right) \subseteq \mathbb{Z} \cdot(K, L, M)$. As remarked in (a), this has the consequence that we can take $\left(h_{0}, n_{0}, q_{0}\right)=(0,0,0)$ in (13.1) and then the definition of the $p_{i}$ implies (in all cases $\mathrm{A}, \mathrm{B}$, see below) that $f_{i j} \neq 0$ and that the quotients $f_{i 1}^{2} /\left(f_{i 0} f_{i 2}\right)$ differ only by roots of unity, hence we get the additional property

$$
\left|\frac{f_{i 1}^{2}}{f_{i 0} f_{i 2}}\right| \quad \text { is constant for } i=1,2,3 .
$$

Comparison of the equations for $x$ and $x^{2 l-1}$ gives $\left(f_{10} / f_{12}\right)^{l-1}=\left(f_{20} / f_{22}\right)^{l-1}$. Doing the same for $x^{2}$ and $x^{2 l-2}$ results in $\left(f_{10} / f_{12}\right)^{l-2}=\left(f_{20} / f_{22}\right)^{l-2}$. Hence

$$
\frac{f_{10}}{f_{12}}=\frac{f_{20}}{f_{22}} .
$$

For $x^{3}$ the equation is (putting $f_{31}^{*}=f_{31}$ for $l=3$ and 0 otherwise)

$$
\begin{aligned}
& (l-1) f_{10}^{l-3}\left(\frac{l-2}{2} f_{11}^{2}+f_{10} f_{12}\right) \\
& \quad+l(l-1) f_{20}^{l-3} f_{21}\left(\frac{l-2}{6} f_{21}^{2}+f_{20} f_{22}\right)+f_{31}^{*}=0 .
\end{aligned}
$$

For $l>3$ this can be combined with the equation for $x$ and yields (after simplification, using (13.4)) $\left(f_{21} / f_{20}\right)^{2}=3\left(f_{11} / f_{10}\right)^{2}$, incompatible with (13.3) and (13.4).

For $l=3$, we consider the equations for $1, x, x^{3}$. Taking the determinant (built of the parts of the three equations corresponding to $p_{1}, p_{2}, p_{3}$ ) gives after simplification

$$
f_{10}^{2} f_{21}^{3} f_{30}-3 f_{11}^{2} f_{20}^{2} f_{21} f_{30}-f_{10}^{2} f_{20}^{3} f_{31}=0 .
$$

This can be rewritten as

$$
\left(\frac{f_{21}}{f_{20}}\right)^{2}-3\left(\frac{f_{11}}{f_{10}}\right)^{2}=\frac{f_{31}}{f_{30}} \frac{f_{20}}{f_{21}} .
$$


Similarly, from the equations for $x, x^{2}$ we get

$$
2 \frac{f_{11}}{f_{10}} \frac{f_{20}}{f_{21}}-1=\frac{f_{20} f_{22}}{f_{21}^{2}} .
$$

Finally, the equations for $x^{0}$ and $x^{6}$ give

$$
\frac{f_{30}}{f_{32}}=\left(\frac{f_{20}}{f_{22}}\right)^{3} \text {. }
$$

In Case A, by (13.1) we have $a^{-1} p_{1}(x)=b^{-1} p_{2}(x)=f_{1}+f_{2} \zeta_{2} x+f_{3} \zeta_{3} x^{2}$ and $c^{-1 / 3} p_{3}(x)=f_{1}+f_{2} \zeta_{2}^{3} x+f_{3} \zeta_{3}^{2} x^{2}$. Then (13.8) gives $f_{1}^{2}=f_{3}^{2},(13.7)$ : $1=\frac{f_{1} f_{3} \zeta_{3}}{f_{2}^{2} \zeta_{2}}$ and (13.6): $(-2)\left(\frac{f_{2} \zeta_{2}}{f_{1}}\right)^{2}=\zeta_{2}^{2}$, which is impossible.

Case Bba transfers to $a^{-1} p_{1}(x)=f_{3} \zeta_{3}^{-1}+f_{2} \zeta_{2}^{-1} x+f_{1} x^{2}, p_{2}, p_{3}$ as in Case A. Then (13.7) gives $2 \frac{f_{1}}{f_{3}} \zeta_{2}^{-2} \zeta_{3}-1=\frac{f_{1} f_{3}}{f_{2}^{2}} \zeta_{2}^{-2} \zeta_{3}$ and (13.6): $\left(\frac{f_{2}}{f_{1}}\right)^{2} \zeta_{2}^{2}-$ $3\left(\frac{f_{2}}{f_{3}}\right)^{2} \zeta_{2}^{-2} \zeta_{3}^{2}=\zeta_{2}^{2}$. Since (from (13.8)) $f_{1}= \pm f_{3}$, these two equations imply $2 \pm \zeta_{2}^{2} \zeta_{3}^{-1}=1-3 \zeta_{2}^{4} \zeta_{3}^{-2}$, hence $1= \pm \zeta_{2}^{2} \zeta_{3}^{-1}-3 \zeta_{2}^{4} \zeta_{3}^{-2}$, which is impossible. Similarly in Cases Baa, Bab. In the case $k=1, r=3$, identity (12.1) becomes $x p_{1}\left(x^{l-1}\right)+p_{2}(x)^{l}+p_{3}\left(x^{M}\right)^{l / M}=0$ with $l \geq 4,1<M<l$. This can be treated similarly to the case $m=1$ above and is somewhat easier since (13.3) is sufficient.

(g) For $r=2, K>0, v_{2}>0$ (this also excludes Case Bbb for the moment), (12.1) becomes for $m=1$ :

$$
x p_{1}\left(x^{K}\right)^{(l-2) / K}+p_{2}(x)^{l}+p_{3}\left(x^{l}\right)=0
$$

and for $k=1$ :

$$
x p_{1}\left(x^{l-2}\right)+p_{2}(x)^{l}+p_{3}\left(x^{M}\right)^{l / M}=0
$$

where $\operatorname{deg} p_{i}=1$ and $l \geq 4, K<l-2,1<M<l$. Again the method of (f) can be used (and since the degrees are smaller, the computations are much easier).

The exceptional cases where $K=0$ (Properties 10.1 and 10.8) have been discussed in Sections 8 and 9(a). They give non-trivial solutions.

In Case A with $v_{2}=0$ (Property 10.2) and Case Bbb (Property 10.11) only $m=1$ is possible (use Lemma 1 as in (c); there can be no common zeros) and (12.1) becomes $x p_{1}(x)^{l-1}+p_{2}(x)^{l}+p_{3}\left(x^{l}\right)=0$ with $l \geq 3$. Here, $l>3$ can be treated as in (f), and $l=3$ leads to non-trivial solutions discussed in Section 9(b),(c).

\section{References}

[A] S. Ahlgren, The set of solutions of a polynomial-exponential equation, Acta Arith. 87 (1999), 189-207. 
[BMZ] E. Bombieri, J. Mueller and U. Zannier, Equations in one variable over function fields, ibid. 99 (2001), 27-39.

[ESS] H. Evertse, H. P. Schlickewei and W. M. Schmidt, Linear equations in variables which lie in a multiplicative group, Ann. of Math. (2) 155 (2002), 807-836.

[G] S. Grünes, Gleichungen $a u_{h}^{k}+b u_{n}^{l}+c u_{q}^{m}=0$ in linearen rekurrenten Folgen $\left\langle u_{n}\right\rangle$, Dissertation, Wien, 1997.

[L1] M. Laurent, Équations exponentielles-polynômes et suites récurrentes linéaires, Astérisque 147-148 (1987), 121-139, 343-344.

[L2] - Équations exponentielles-polynômes et suites récurrentes linéaires, II, J. Number Theory 31 (1989), 24-53.

[M1] R. C. Mason, Diophantine Equations over Function Fields, London Math. Soc. Lecture Note Ser. 96, Cambridge Univ. Press, Cambridge, 1984.

[M2] - Equations over Function Fields, Lecture Notes in Math. 1068, Springer, Berlin, 1984.

[SS1] H. P. Schlickewei and W. M. Schmidt, Linear equations in members of recurrence sequences, Ann. Scuola Norm. Sup. Pisa Cl. Sci. (4) 20 (1993), 219-246.

[SS2] -, - Equations a $u_{n}^{l}=b u_{m}^{k}$ satisfied by members of recurrence sequences, Proc. Amer. Math. Soc. 118 (1993), 1043-1051.

[SS3] - - - The number of solutions of polynomial-exponential equations, Compositio Math. 120 (2000), 193-225.

[ST] T. N. Shorey and R. Tijdeman, Exponential Diophantine Equations, Cambridge Tracts in Math. 87, Cambridge Univ. Press, Cambridge, 1986.

Institut für Mathematik der Universität Wien

Strudlhofgasse 4

A-1090 Wien, Austria

E-mail: losert@ap.univie.ac.at

Received on 24.6.2003

and in revised form on 31.3.2005 